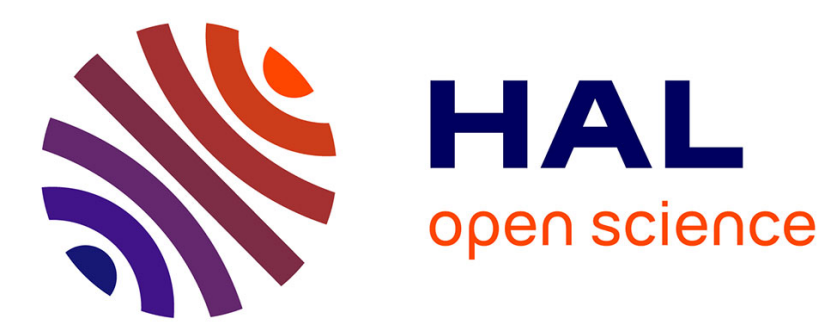

\title{
On a nonlocal system for vegetation in drylands
}

\author{
Matthieu Alfaro, Hirofumi Izuhara, Masayasu Mimura
}

\section{To cite this version:}

Matthieu Alfaro, Hirofumi Izuhara, Masayasu Mimura. On a nonlocal system for vegetation in drylands. Journal of Mathematical Biology, 2018, 77 (6-7), pp.1761-1793. 10.1007/s00285-018-1215-0 . hal-01982126

\section{HAL Id: hal-01982126 \\ https://hal.science/hal-01982126}

Submitted on 15 Jan 2019

HAL is a multi-disciplinary open access archive for the deposit and dissemination of scientific research documents, whether they are published or not. The documents may come from teaching and research institutions in France or abroad, or from public or private research centers.
L'archive ouverte pluridisciplinaire $\mathbf{H A L}$, est destinée au dépôt et à la diffusion de documents scientifiques de niveau recherche, publiés ou non, émanant des établissements d'enseignement et de recherche français ou étrangers, des laboratoires publics ou privés. 


\title{
On a nonlocal system for vegetation in drylands
}

\author{
Matthieu Alfaro*, Hirofumi Izuhara ${ }^{\dagger}$ and Masayasu Mimura ${ }^{\ddagger \S}$
}

January 31, 2018

\section{Contents}

1 Introduction $\quad 1$

2 Well-posedness of the nonlocal system $\quad 5$

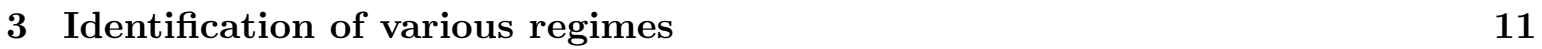

4 Steady states 12

4.1 Constant steady states . . . . . . . . . . . . . . . . . . . . . . 13

4.2 Global structure of stationary solutions . . . . . . . . . . . . . . . . 15

4.3 Global structure of stationary solutions for other kernels . . . . . . . . . . . 20

4.4 Two dimensional vegetation patterns . . . . . . . . . . . . . . . . 25

\section{Concluding remarks $\quad 28$}

\begin{abstract}
Several mathematical models are proposed to understand spatial patchy vegetation patterns arising in drylands. In this paper, we consider the system with nonlocal dispersal of plants (through a redistribution kernel for seeds) proposed by Pueyo et al. in [27] as a model for vegetation in water-limited ecosystems. It consists in two reaction diffusion equations for surface water and soil water, combined with an integro-differential equation for plants. For this system, under suitable assumptions, we prove well-posedness using the Schauder fixed point theorem. In addition, we consider the stationary problem from the viewpoint of vegetated pattern formation, and show a transition of vegetation patterns when parameter values (rainfall, seed dispersal range, seed germination rate) in the system vary. The influence of the shape of the redistribution kernel is also discussed.
\end{abstract}

\section{Introduction}

In drylands, vegetation patterns with patchiness such as stripes, spots, labyrinths and gaps are observed [2], [4], [6], [14], [28], [30], [37]. The distance between stripes/spots of the

*IMAG, Université de Montpellier, CC051, Place Eugène Bataillon, 34095 Montpellier Cedex 5, France.

${ }^{\dagger}$ Faculty of Engineering, University of Miyazaki, 1-1 Gakuen Kibanadai Nishi, Miyazaki, 889-2192, Japan.

${ }^{\ddagger}$ Faculty of Engineering, Musashino University, 3-3-3 Ariake, Koto-ku, Tokyo 135-8181, Japan

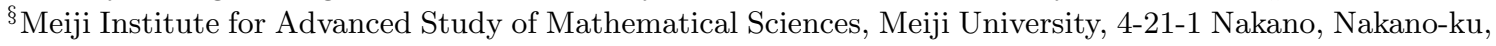
Tokyo 164-8525, Japan. 
vegetation patterns is wide-ranging from tens of centimeters to hundreds of meters, depending on plant species. Moreover, it is also reported that vegetation patterns observed in fields are independent of soil type and specific plant species. Figure 1 shows typical vegetation patterns in water-limited ecosystems. It is said that the patchiness of vegetation in water-limited
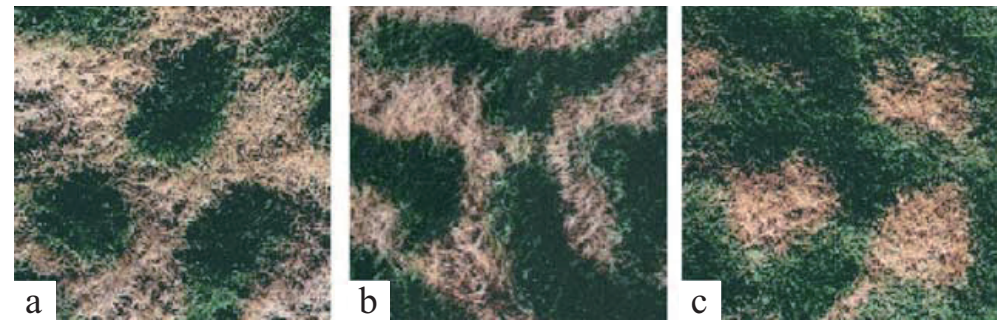

Figure 1: Vegetation patterns of the perennial grass Paspalum varinatum in Israel (200mm mean annual rainfall). (a) spots. (b) stripes. (c) gap. The distance between spots/stripes is of the order of $15 \mathrm{~cm}$. Reproduced with permission from [10].

ecosystems is formed in a self-organized way [21], [30], [31], [37] and an important signal to catastrophic shifts for desertification [30], [32]. In order to understand such self-organized vegetation pattern formation, a theoretical approach is required, see [2], [19], [20] for instance. For a few decades, a lot of mathematical models to describe the vegetation pattern formation in drylands have been developed. Klausmeier [16] focused on two important factors, plant density and water concentration, to describe stripe vegetation patterns formed on slopes and proposed a two component reaction-diffusion-advection system. We refer to [33], [34] and the references therein for studies using Klausmeier model. Based on the Klausmeier model, a lot of improvements have been performed. The authors in [11], [26] focus on three factors, plant density, surface water and soil water. The proposed models are reaction-diffusion systems, which include only local effects. Also, the authors in [7], [8] proposed a three-component model of plant density, surface water and soil water, which includes a nonlocal effect described as water uptake by laterally extended roots. Moreover, the existence of solutions for the system proposed in [7], [8] was proved in [9]. Many researchers suggest that the vegetation patterns in water-limited ecosystems are generated as a result of the diffusion-induced instability (the Turing instability) [11], [13], [21], [26], [29], [31]. In particular, the work [15] discuss the diversity of vegetation patterns in drylands through interplay between Turing mechanisms.

On the other hand, each plant establishes seed dispersal that contributes to the spreading. In describing seed dispersal, nonlocal terms are more applicable for population dynamics of seed dispersed plants than the diffusion term [1], [36]. In this context, Pueyo et al. [27] proposed a new mathematical model with three components, plant density and amounts of soil water and surface water. A feature of this model is to contain a nonlocal term for the "plant spreading". It reads

$$
\begin{aligned}
\frac{\partial P}{\partial t} & =c_{P} g_{P_{\max }} \frac{W}{W+k_{1}} P-\left(d_{P}+f\right) P+r_{c} \frac{W}{W+k_{1}} g_{S} \int_{\Omega} f P(t, y) \Phi(x, y) d y, \\
\frac{\partial W}{\partial t} & =\alpha U \frac{P+k_{2} w_{U}}{P+k_{2}}-g_{P_{\max }} \frac{W}{W+k_{1}} P-r_{W} W+d_{W} \Delta W, \\
\frac{\partial U}{\partial t} & =r_{\text {rain }}-\alpha U \frac{P+k_{2} w_{U}}{P+k_{2}}+d_{U} \Delta U,
\end{aligned}
$$


where $P(t, x), W(t, x)$ and $U(t, x)(t>0, x \in \Omega)$ respectively represent plant density, soil water and surface water at time $t$ and position $x$. Here, $\Omega$ is a connected bounded open set of $\mathbb{R}^{N}$, with smooth boundary $\partial \Omega$. All the parameter values are positive constants. The equation for the plant density $P$ consists of plant growth and loss, and establishment after germination of seeds. The plant growth depends on amount of soil water $W(t, x)$, described as Michaelis-Mentan kinetics and the plant loss is at a constant rate. The integral term $\int_{\Omega} f P(t, y) \Phi(x, y) d y$ in the third term of the right hand side in the equation for $P$ means seed density at time $t$ and position $x$, that is seeds are produced by the plant $P$ at a constant rate $f$. Here, the kernel $\Phi$, which is often called redistribustion kernel, is a probability density function, therefore, we assume that $\int_{\Omega} \Phi(x, y) d y=1$ for any $x \in \Omega$. In other words, the integral term describes the distribution of seeds produced by plants. We call this term seed dispersal in this paper. In addition, the term $r_{c} \frac{W}{W+k_{1}} g_{S}$ in front of the integral term means an establishment rate for seedlings, which also depends on soil water. Thus the third term describes supply of biomass through germination of seeds. The second and third equations describe the dynamics of water, where water is divided into two stages: soil water and surface water. The constant $r_{\text {rain }}$ is rainfall and the term $-\alpha U \frac{P+k_{2} w_{U}}{P+k_{2}}$ represents an infiltration rate from ground into subsurface, which depends on the plant density: plenty of biomass facilitates the infiltration of water $\left(w_{U}<1\right)$. The terms $-g_{P_{\max }} \frac{W}{W+k_{1}} P$ and $-r_{W} W$ in the equation for $W$ mean the losses of soil water due to uptake by plants and due to drainage and evaporation, respectively. Finally, the dispersal of soil water and surface water is described by linear diffusion. Actually, surface water flow is not a linear diffusion process and can be modeled by using shallow water theory [7], [18]. However, the results obtained with such a nonlinear diffusion process are qualitatively similar to those obtained in the linear case. This justifies the use of a linear diffusion process as surface water flow in (1).

In order to reduce the number of parameters, we use the following change of variables (nondimensionalization):

$$
\begin{aligned}
& \tilde{P}(\tilde{t}, \tilde{x})=\frac{1}{k_{2}} P(t, x), \quad \tilde{W}(\tilde{t}, \tilde{x})=\frac{1}{k_{1}} W(t, x), \quad \tilde{U}(\tilde{t}, \tilde{x})=\frac{\alpha}{g_{P_{\max }} k_{2}} U(t, x), \\
& \tilde{\Phi}(\tilde{x}, \tilde{y})=\left(\frac{d_{U} k_{1}}{g_{P_{\max }} k_{2}}\right)^{N / 2} \Phi(x, y), \\
& \tilde{t}=\frac{g_{P_{\max }} k_{2}}{k_{1}} t, \quad \tilde{x}=\sqrt{\frac{g_{P_{\max }} k_{2}}{d_{U} k_{1}}} x, \quad \tilde{y}=\sqrt{\frac{g_{P_{\max }} k_{2}}{d_{U} k_{1}}} y, \\
& \tilde{\Omega}=\left\{\sqrt{\frac{g_{P_{\max }} k_{2}}{d_{U} k_{1}}} z, z \in \Omega\right\}, \\
& a=\frac{c_{P} k_{1}}{k_{2}}, \quad b=\frac{\left(d_{P}+f\right) k_{1}}{g_{P_{\max } k_{2}}}, \quad c=\frac{r_{c} g_{S} f k_{1}}{g_{P_{\max } k_{2}}}, \quad d=\frac{d_{W}}{d_{U}}, \quad e_{0}=\frac{r_{W} k_{1}}{g_{P_{\max }} k_{2}}, \\
& r=\frac{\alpha r_{\operatorname{rain}} k_{1}}{\left(g_{P_{\max }} k_{2}\right)^{2}}, \quad g=\frac{\alpha k_{1}}{g_{P_{\max }} k_{2}} .
\end{aligned}
$$


Then, (1) is recast into

$$
\begin{aligned}
\frac{\partial P}{\partial t} & =a \frac{W}{W+1} P-b P+c \frac{W}{W+1} \int_{\Omega} P(t, y) \Phi(x, y) d y, \\
\frac{\partial W}{\partial t} & =d \Delta W+U \frac{P+w_{U}}{P+1}-\frac{W}{W+1} P-e_{0} W, \quad t>0, x \in \Omega \\
\frac{\partial U}{\partial t} & =\Delta U+r-g U \frac{P+w_{U}}{P+1}
\end{aligned}
$$

where we have dropped the tildes.

In this paper, since we impose the Neumann boundary conditions for surface water and soil water, the corresponding redistribution kernel is needed. Precisely, we assume the following.

Assumption 1.1 (Redistribution kernel) Kernel $\Phi: \bar{\Omega} \times \bar{\Omega} \rightarrow\left[0, \Phi_{\text {max }}\right]$ is continuous, and satisfies

$$
\Phi(x, y) \geq \Phi_{0}>0 \quad \text { when }|x-y| \leq \delta_{\Phi},
$$

for some constants $\Phi_{0}>0, \delta_{\Phi}>0$, and, for all $x \in \bar{\Omega}$,

$$
\int_{\Omega} \Phi(x, y) d y=1
$$

together with

$$
D_{y} \Phi(x, y) \cdot n_{y}=0 \quad \text { for all } y \in \partial \Omega,
$$

where $n_{y}$ is an outward unit normal vector to $\partial \Omega$ at point $y$.

For instance, in one space dimension $(0, L)$, one can simply think of

$$
k(x, y):=\frac{1}{\xi} e^{-\eta(x-y)^{2}}
$$

and

$$
\Phi(x, y)=\sum_{i=-\infty}^{\infty}(k(x, y+2 i L)+k(x, 2 i L-y))
$$

where the constant $\xi$ is adjusted so that $\int_{0}^{L} \Phi(x, y) d y=1$ (see [12]). To perform numerics in Section 4, we shall use the approximation

$$
\Phi_{\text {approx }}(x, y)=k(x, y)+k(x,-y)+k(x, 2 L-y) .
$$

Figure 2 shows a graph of the kernel $\Phi_{\text {approx }}(x, y)$. The derivative conditions on the boundary are approximately satisfied. From the seed dispersal point of view, the works [3], [22], [23], [24] discuss suitable redistribution kernels. In subsection 4.3, we consider other kernels than (5), allowing in particular bimodal shapes.

In [27], Pueyo et al. proposed the model (1), and they discussed the relation between spatial patterns and biomass, and the effects of plant dispersal strategies, fecundity and establishment ability. In this paper, we discuss relations between spatial vegetation patterns and four parameter in the system: the rainfall rate, the seed dispersal range, the seed germination rate and the kernel shape. On the basis of these discussions, we first argue the existence 


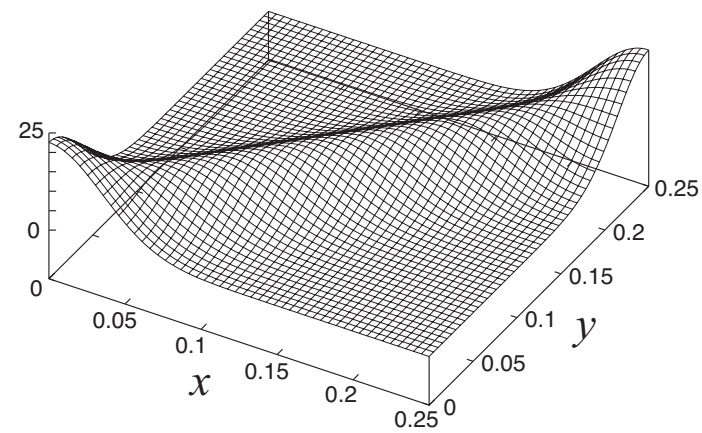

Figure 2: Graph of the kernel $\Phi_{\text {approx }}(x, y)$ with $k(x, y)=\frac{1}{\xi} e^{-\eta(x-y)^{2}}$. The horizontal axis and the depth mean $x$ and $y$, respectively. Here, we set $\xi=0.088587, \eta=400$ and $L=0.25$.

and uniqueness of a solution to the system (2) under the Neumann boundary conditions and suitable initial functions.

The paper is organized as follows. In Section 2, we prove the well-posedness of the nonlocal system (2) by using the Schauder fixed point theorem and a comparison principle argument. In Section 3 we identify, depending on the values of parameters, three regimes: hyper-arid and arid ecosystems both leading to extinction of plants, in contrast with semi-arid ecosystems possibly leading to patchy patterns. In Section 4, we perform computer-aided analysis to shed light on bifurcations from constant to heterogeneous steady states, that are natural candidates to describe patchiness. Besides, the influence of the variation of some parameter values on two-dimensional vegetation patterns are numerically illustrated. Finally, we give some concluding remarks in Section 5 .

\section{Well-posedness of the nonlocal system}

In this section we prove, under suitable assumptions, the well-posedness of the nonlocal system (2) equipped with the no flux boundary conditions for surface water and soil water.

For this analysis, let us use the small letters $u, w, p$, and then consider

$$
\begin{cases}\partial_{t} u-\Delta u=r-g H(p) u & t>0, x \in \Omega, \\ \partial_{t} w-d \Delta w+e_{0} w=H(p) u-K(w) p & t>0, x \in \Omega, \\ \partial_{t} p+b p=a K(w) p+M(w) c \int_{\Omega} \Phi(x, y) p(t, y) d y & t>0, x \in \Omega, \\ (u(0, x), w(0, x), p(0, x))=\left(u_{0}(x), w_{0}(x), p_{0}(x)\right) & x \in \Omega, \\ \left(\frac{\partial u}{\partial \nu}(t, x), \frac{\partial w}{\partial \nu}(t, x)\right)=(0,0) & t>0, x \in \partial \Omega,\end{cases}
$$

where $r, g, d, e_{0}, b, a$ and $c$ are positive constants. To stick with (2) we could select $H(p)=$ $\frac{p+w_{U}}{p+1}, K(w)=M(w)=\frac{w}{w+1}$, but we allow more profiles by only making the following assumptions. 
Assumption 2.1 (Nonlinear functions) Functions $H, K, M$ are increasing and locally Lipschitz continuous on $[0, \infty)$ and satisfy, for some constants $H^{+}, K^{+}, M^{+}$,

$$
\begin{gathered}
0<H(0) \leq H(p) \leq H^{+} \quad \text { for all } p \geq 0, \\
0=K(0)<K(w) \leq K^{+} \quad \text { for all } w>0, \\
0=M(0)<M(w) \leq M^{+} \quad \text { for all } w>0 .
\end{gathered}
$$

As far as initial conditions are concerned, our assumptions are the following.

Assumption 2.2 (Initial conditions) The initial data $\left(u_{0}, w_{0}, p_{0}\right)$ are smooth, nonnegative, $p_{0} \not \equiv 0$, and satisfy the expected compatibility conditions that is $\left(\frac{\partial u_{0}}{\partial \nu}(x), \frac{\partial w_{0}}{\partial \nu}(x)\right)=(0,0)$, for all $x \in \partial \Omega$.

We start with some useful a priori estimates. In the sequel, for $T>0$, we define $Q_{T}:=$ $(0, T) \times \Omega$.

Lemma 2.3 (A priori estimates) Let Assumptions 2.1, 2.2 and 1.1 hold. Then, for any $T>0$, there is a constant $C>0$ such that any nonnegative solution $(u, w, p) \in C\left(\overline{Q_{T}}\right)^{3}$ of the nonlocal system (6) satisfies

$$
0 \leq u(t, x), w(t, x), p(t, x) \leq C \quad \text { for all }(t, x) \in \overline{Q_{T}} .
$$

Proof. - Notice that the constants 0 and $\frac{r}{g H(0)}$ are sub- and super-solutions for equation $\partial_{t} u-\Delta u=r-g H(p) u$, therefore it follows from the parabolic maximum principle that

$$
0 \leq u(t, x) \leq C_{u}:=\max \left(\frac{r}{g H(0)},\left\|u_{0}\right\|_{L^{\infty}(\Omega)}\right),
$$

and $u(t, x)>0$ as soon as $t>0, x \in \Omega$, thanks to the strong maximum principle.

- The above control on $u$ then implies that the constants 0 and $H^{+} C_{u} / e_{0}$ are sub- and super-solutions for equation $\partial_{t} w-d \Delta w+e_{0} w=H(p) u-K(w) p$, and therefore

$$
0 \leq w(t, x) \leq C_{w}:=\max \left(\frac{H^{+} C_{u}}{e_{0}},\left\|w_{0}\right\|_{L^{\infty}(\Omega)}\right)
$$

and $w(t, x)>0$ as soon as $t>0, x \in \Omega$, thanks to the strong maximum principle.

- To prove the nonnegativity of $p$, we write

$$
\partial_{t} p+(b-a K(w(t, x))) p=\theta(t, x):=M(w(t, x)) c \int_{\Omega} \Phi(x, y) p(t, y) d y
$$

which we regard as a first order ODE (with $x$ playing the role of a parameter), so that we have the implicit formula

$$
p(t, x)=e^{-b t+a \int_{0}^{t} K(w(\tau, x)) d \tau}\left(p_{0}(x)+\int_{0}^{t} e^{b \tau-a \int_{0}^{\tau} K(w(h, x)) d h} \theta(\tau, x) d \tau\right) .
$$


In particular, for any $x \in \bar{\Omega}$, the continuity of $p(\cdot, x), K(w(\cdot, x))$ and $M(w(\cdot, x))$ on $[0, T]$ enforces the partial application $t \mapsto p(t, x)$ to belong to $C^{1}([0, T])$. We claim that, for any $x \in \bar{\Omega}$, we can define

$$
t_{x}:=\sup \{t \in(0, T]: 0<\tau<t \Longrightarrow p(\tau, x)>0\} \in(0, T] .
$$

Indeed, if $p(0, x)=p_{0}(x)>0$ this is trivial. On the other hand, if $p(0, x)=p_{0}(x)=0$, evaluating $(12)$ at point $(0, x)$ yields

$$
\partial_{t} p(0, x)=M\left(w_{0}(x)\right) c \int_{\Omega} \Phi(x, y) p_{0}(y) d y,
$$

which is strictly positive - in view of $p_{0} \geq 0, p_{0} \not \equiv 0$ and (3) - which also validates definition (14). The function $x \mapsto t_{x}$ being lower semi continuous on the compact $\bar{\Omega}$, we can define

$$
T^{*}:=\min _{x \in \bar{\Omega}} t_{x} \in(0, T] .
$$

Let us assume, by way of contradiction, that $T^{*}<T$. Then, for a $x^{*}$ such that $T^{*}=t_{x^{*}}$, we have $p\left(T^{*}, x^{*}\right)=0$ so that, by evaluating (13) at point $\left(T^{*}, x^{*}\right)$, we get that $p_{0}\left(x^{*}\right)=0$ and $\theta\left(\tau, x^{*}\right)=M\left(w\left(\tau, x^{*}\right)\right) c \int_{\Omega} \Phi\left(x^{*}, y\right) p(\tau, y) d y=0$ for all $0 \leq \tau \leq T^{*}$. In view of (9), $M\left(w\left(\tau, x^{*}\right)\right)>0$ as soon as $\tau>0$ so that

$$
\int_{\Omega} \Phi\left(x^{*}, y\right) p(\tau, y) d y=0 \quad \text { for all } 0<\tau \leq T^{*} .
$$

This, combined with assumption $(3)$, enforces $p(\tau, y)=0$ for all $(\tau, y)$ in the cylinder $\left[0, T^{*}\right] \times$ $B\left(x^{*}, \delta_{\Phi}\right)$. A next point $x^{* *}$ is taken from inside of $B\left(x^{*}, \delta_{\Phi}\right) \backslash B\left(x^{*}, \frac{1}{2} \delta_{\Phi}\right)$. By repeating the above procedure, we arrive at $p \equiv 0$ in $\left[0, T^{*}\right] \times B\left(x^{* *}, \delta_{\Phi}\right)$. By repeating a finite number of times this, we get $p \equiv 0$ on the compact region $\overline{Q_{T^{*}}}$, which contradicts $p_{0} \not \equiv 0$. As a result, $T^{*}=T$ and the nonnegativity of $p$ is proved. We have even proved that $p(t, x)>0$ as soon as $t>0, x \in \bar{\Omega}$.

- It remains to bound $p$ from above. The equation for $p$ in (6) yields

$$
\partial_{t} p(t, x) \leq\left(a K^{+}-b\right) p(t, x)+M^{+} c \int_{\Omega} \Phi(x, y) p(t, y) d y .
$$

Defining the total mass of plants $P(t):=\int_{\Omega} p(t, x) d x$, integrating the above inequality over $x \in \Omega$, using Fubini-Tonelli theorem and (4) we obtain $P^{\prime}(t) \leq \beta P(t)$, where

$$
\beta:=a K^{+}-b+M^{+} c=: \delta+M^{+} c .
$$

It therefore follows that $P(t) \leq P(0) e^{\beta t}$ which we plug into (15) to get the differential inequality

$$
\partial_{t} p(t, x) \leq \delta p(t, x)+M^{+} c \Phi_{\max } P(0) e^{\beta t}, \quad p(0, x)=p_{0}(x) .
$$

Since $\left(p_{0}(x)-\Phi_{\max } P(0)\right) e^{\delta t}+\Phi_{\max } P(0) e^{\beta t}$ is the solution of the equation associated naturally with $(17)$, we get by comparison that

$$
\begin{aligned}
p(t, x) & \leq\left(p_{0}(x)-\Phi_{\max } P(0)\right) e^{\delta t}+\Phi_{\max } P(0) e^{\beta t} \\
& \leq\left(\left\|p_{0}\right\|_{L^{\infty}(\Omega)}+\Phi_{\max } P(0)\right) e^{\beta t} \\
& \leq\left(\left\|p_{0}\right\|_{L^{\infty}(\Omega)}+\Phi_{\max } P(0)\right) \max \left(1, e^{\beta T}\right)=: C_{p},
\end{aligned}
$$

which concludes the proof of the lemma. 
Remark 2.4 (Hyper-arid ecosytems) If the constant $\beta=a K^{+}-b+M^{+}$c turns out to be negative, then there is $p_{\max }>0$ such that, for all $x \in \Omega$,

$$
p(t, x) \leq p_{\max } e^{\beta t} \rightarrow 0, \quad \text { as } t \rightarrow \infty,
$$

that is an exponentially fast extinction of plants.

We now prove the well-posedness of problem (6) by using the Schauder fixed point theorem.

Theorem 2.5 (Well-posedness of the nonlocal system) Let Assumptions 2.1, 2.2 and 1.1 hold. Then, for any $T>0$, the nonlocal problem (6) has a unique nonnegative solution $(u, w, p)$ in $C^{\frac{1+\alpha}{2}, 1+\alpha}\left(\overline{Q_{T}}\right) \times C^{\frac{1+\alpha}{2}, 1+\alpha}\left(\overline{Q_{T}}\right) \times\left(C\left(\overline{Q_{T}}\right) \cap C_{t}^{1}\left(\overline{Q_{T}}\right)\right)$ for any $0<\alpha<1$.

Proof. Let $T>0$ be given. Let us define a convex and closed subset of $X:=C\left(\overline{Q_{T}}\right)^{3}$ by

$$
A:=\left\{(u, w, p) \in X: 0 \leq u, w, p \leq C,(u, w, p)(0, x)=\left(u_{0}, w_{0}, p_{0}\right)(x), P(t) \leq P(0) e^{\beta t}\right\},
$$

where $C:=\max \left(C_{u}, C_{w}, C_{p}\right)$ (see (10), (11), (18) in proof of Lemma 2.3), $P(t):=\int_{\Omega} p(t, x) d x$ and $\beta$ is defined in (16). Let $(u, w, p) \in A$ be given.

- We denote by $\bar{u}$ the solution of the linear (observe that the initially given $p$ is plugged in the right hand side member) parabolic problem

$$
\begin{cases}\partial_{t} \bar{u}-\Delta \bar{u}=r-g H(p) \bar{u} & t>0, x \in \Omega \\ \bar{u}(0, x)=u_{0}(x) & x \in \Omega \\ \frac{\partial \bar{u}}{\partial \nu}(t, x)=0 & t>0, x \in \partial \Omega\end{cases}
$$

We have $0 \leq \bar{u} \leq C_{u}$ (see proof of Lemma 2.3) and it follows from standard parabolic estimates (see e.g. [17]) that

$$
\|\bar{u}\|_{C^{\frac{1+\alpha}{2}, 1+\alpha}\left(\overline{Q_{T}}\right)} \leq \overline{C_{u}},
$$

where $\overline{C_{u}}$ does not depend on $(u, w, p) \in A$ but only on $C, T,|\Omega|, r, g, H^{+}$.

- We also denote by $\bar{w}$ the solution of the nonlinear parabolic problem (observe that the $\bar{u}$ obtain previously - and not the initially given $u$ - is plugged in the right-hand side member to insure that $C_{w}$ remains a super-solution)

$$
\begin{cases}\partial_{t} \bar{w}-d \Delta \bar{w}+e_{0} \bar{w}=H(p) \bar{u}-K(\bar{w}) p & t>0, x \in \Omega \\ \bar{w}(0, x)=w_{0}(x) & x \in \Omega \\ \frac{\partial \bar{w}}{\partial \nu}(t, x)=0 & t>0, x \in \partial \Omega .\end{cases}
$$

Notice that the existence of $\bar{w}$ is guaranteed (see [35, Chapter 14]) by the a priori estimate $0 \leq \bar{w} \leq C_{w}$ (see proof of Lemma 2.3). It follows from standard parabolic estimates that

$$
\|\bar{w}\|_{C^{\frac{1+\alpha}{2}, 1+\alpha}\left(\overline{Q_{T}}\right)} \leq \overline{C_{w}}
$$

where $\overline{C_{w}}$ does not depend on $(u, w, p) \in A$ but only on $C, T,|\Omega|, d, e_{0}, H^{+}, K^{+}$.

- Last we denote by $\bar{p}$ the solution of the first order ODE, with $x$ playing the role of a parameter, (observe that the initially given $p$ and the previously obtained $\bar{w}-$ and not the 
initially given $w$ - are plugged in the right hand side member; see below for an explanation of this choice)

$$
\left\{\begin{array}{l}
\partial_{t} \bar{p}+(b-a K(\bar{w}(t, x))) \bar{p}=\theta(t, x):=M(\bar{w}(t, x)) c \int_{\Omega} \Phi(x, y) p(t, y) d y \\
\bar{p}(0, x)=p_{0}(x)
\end{array}\right.
$$

that is

$$
\bar{p}(t, x)=e^{-b t+a \int_{0}^{t} K(\bar{w}(\tau, x)) d \tau}\left(p_{0}(x)+\int_{0}^{t} e^{b \tau-a \int_{0}^{\tau} K(\bar{w}(h, x)) d h} \theta(\tau, x) d \tau\right) .
$$

The nonnegativity of $p$ implies that of $\bar{p}$. Having inserted the control $P(t) \leq P(0) e^{\beta t}$ in the definition of the set $A$ allows to recover $\bar{p} \leq C_{p} \leq C$. Indeed, the analogous of (15), that is

$$
\partial_{t} \bar{p}(t, x) \leq\left(a K^{+}-b\right) \bar{p}(t, x)+M^{+} c \int_{\Omega} \Phi(x, y) p(t, y) d y,
$$

combined with $P(t) \leq P(0) e^{\beta t}$ implies the analogous of (17) for $\bar{p}$, which implies $\bar{p} \leq C_{p}$ as in the proof of Lemma 2.3. On the other hand, we need to show that the control $\bar{P}(t):=$ $\int_{\Omega} \bar{p}(t, x) d x \leq P(0) e^{\beta t}$ has not been lost. Integrating (25) over $x \in \Omega$, using Fubini-Tonelli theorem and (4) we obtain

$$
\bar{P}^{\prime}(t) \leq\left(a K^{+}-b\right) \bar{P}(t)+M^{+} c P(t) \leq\left(a K^{+}-b\right) \bar{P}(t)+M^{+} c P(0) e^{\beta t} .
$$

By the definition of $\beta$ in (16), $P(0) e^{\beta t}$ solves the equation associated naturally with (26), so that by comparison we get the desired estimate $\bar{P}(t) \leq P(0) e^{\beta t}$. We now prove that

$$
\{\bar{p}:(u, w, p) \in A\} \text { is relatively compact in } C\left(\overline{Q_{T}}\right),
$$

using Arzéla-Ascoli theorem. The uniform boundedness is already known and the equicontinuity follows from the following two facts. First, $(23)$ provides a uniform (w.r.t. $(u, w, p) \in A$, $\left.(t, x) \in \overline{Q_{T}}\right)$ bound for $\left|\partial_{t} \bar{p}(t, x)\right|$, which settles the case of the time variable. Secondly, the case of the space variable directly follows from the expression (24) and the uniform continuity of $M, K, \Phi$ on compact sets, the uniform continuity of $p_{0}$ on $\bar{\Omega}$ and the uniform (w.r.t. $(u, w, p) \in A$ ) bound $(22)$ (notice that this is where we would have faced a problem if we had plugged the initially given $w$, which is only continuous, rather than $\bar{w}$ into (23)).

- Hence, we are equipped with the continuous operator

$$
\mathcal{L}:(u, w, p) \in A \mapsto(\bar{u}, \bar{w}, \bar{p}) \in A,
$$

with $A$ a convex and closed subset of the Banach space $X$. Moreover it follows from estimates $(20),(22)$ and $(27)$ that $\mathcal{L}(A)$ is relatively compact. Therefore, by the Schauder fixed point theorem, the operator $\mathcal{L}$ has a fixed point, that is a solution of the nonlocal problem (6).

- Last, uniqueness follows from an adaptation of classical procedures for local system. If $\left(u_{1}, w_{1}, p_{1}\right)$ and $\left(u_{2}, w_{2}, p_{2}\right)$ are two solutions then, in virtue of the a priori estimate Lemma 2.3 , all components lie in the interval $[0, C]$ on which the nonlinear functions $H, K$ and $M$ are Lipschitz continuous. Subtract the equation for $u_{2}$ from that for $u_{1}$, multiply the result by $u:=u_{1}-u_{2}$ and then integrate over the domain $\Omega$. Proceed similarly for other equations, add the three estimates to collect

$$
\frac{d}{d t} \int_{\Omega}\left(u^{2}+w^{2}+p^{2}\right)(t, x) d x \leq C s t \int_{\Omega}\left(u^{2}+w^{2}+p^{2}\right)(t, x) d x
$$


where $C$ st is a positive constant. Then the Gronwall lemma yields $u \equiv w \equiv p \equiv 0$. We skip the details but uniqueness actually also follows from the following stronger property, whose proof is given.

Proposition 2.6 (Comparison principle) Let $\left(u_{*}, w_{*}, p_{*}\right) \geq(0,0,0)$ and $\left(u^{*}, w^{*}, p^{*}\right) \geq$ $(0,0,0)$ be such that

$$
\left\{\begin{array}{c}
\partial_{t} u_{*}-\Delta u_{*}-r+g H\left(p^{*}\right) u_{*} \\
\leq \partial_{t} u^{*}-\Delta u^{*}-r+g H\left(p_{*}\right) u^{*} \\
\partial_{t} w_{*}-d \Delta w_{*}+e_{0} w_{*}-H\left(p_{*}\right) u_{*}+K\left(w_{*}\right) p^{*} \\
\leq \partial_{t} w^{*}-d \Delta w^{*}+e_{0} w^{*}-H\left(p^{*}\right) u^{*}+K\left(w^{*}\right) p_{*} \\
\partial_{t} p_{*}+b p_{*}-a K\left(w_{*}\right) p_{*}-M\left(w_{*}\right) c \int_{\Omega} \Phi(x, y) p_{*}(t, y) d y \\
\leq \partial_{t} p^{*}+b p^{*}-a K\left(w^{*}\right) p^{*}-M\left(w^{*}\right) c \int_{\Omega} \Phi(x, y) p^{*}(t, y) d y
\end{array}\right.
$$

together with $\left(u_{*}, w_{*}, p_{*}\right)(0, \cdot) \leq\left(u^{*}, w^{*}, p^{*}\right)(0, \cdot)$ in $\Omega$, and $\left(\frac{\partial u_{*}}{\partial \nu}, \frac{\partial w_{*}}{\partial \nu}\right) \leq\left(\frac{\partial u^{*}}{\partial \nu}, \frac{\partial w^{*}}{\partial \nu}\right)$ in $(0, T) \times$ $\partial \Omega$. Then

$$
\left(u_{*}, w_{*}, p_{*}\right) \leq\left(u^{*}, w^{*}, p^{*}\right) \quad \text { in } \overline{Q_{T}} .
$$

Proof. Define functions $u:=u_{*}-u^{*}, w:=w_{*}-w^{*}, p:=p_{*}-p^{*}$. Denote by $L>0$ an upper bound for the Lipschitz constants of $H, K$ and $M$ on the interval $\left[0, C_{\max }\right]$, in which $u_{*}, w_{*}$, $p_{*}, u^{*}, w^{*}, p^{*}$ take their values. Multiplying the first inequality of $(28)$ by $u^{+}:=\max (u, 0)$, integrating over $Q_{t}=(0, t) \times \Omega$, using $u^{+}(0, \cdot) \equiv 0$, using $\int_{\Omega} u^{+} \Delta u d x \leq-\int_{\Omega}\left|\nabla u^{+}\right|^{2} d x$ thanks to $\frac{\partial u}{\partial \nu}(t, \cdot) \leq 0$ on $\partial \Omega$, we get

$$
\frac{1}{2} \int_{\Omega}\left(u^{+}\right)^{2}(t, x) d x \leq \int_{0}^{t} \int_{\Omega} g\left(H\left(p_{*}\right) u^{*}-H\left(p^{*}\right) u_{*}\right) u^{+} d x d \tau .
$$

We distinguish three different cases to estimate the integrand in the right hand side member above: if $u_{*} \leq u^{*}$ it is zero; if $u_{*}>u^{*}$ and $p^{*} \geq p_{*}$ it is non positive by the monotonicity of $H$ and the nonnegativity of $u_{*}$ and $u^{*}$; if $u_{*}>u^{*}$ and $p^{*}<p_{*}$ then it is controlled by $g\left(L C_{\max } p^{+}+H^{+} u^{+}\right) u^{+}$, since in this case $|p|=p^{+}$, and hence by $C_{1}\left(\left(p^{+}\right)^{2}+\left(u^{+}\right)^{2}\right)$. Here, $p^{+}$is defined as $p^{+}:=\max (p, 0)$. As a result we have

$$
\int_{\Omega}\left(u^{+}\right)^{2}(t, x) d x \leq C_{1} \int_{0}^{t} \int_{\Omega}\left(\left(u^{+}\right)^{2}+\left(p^{+}\right)^{2}\right)(\tau, x) d x d \tau .
$$

Similar arguments (multiply by $w^{+}:=\max (w, 0)$, integrate, distinguish cases, use monotonicity and positivity) for the second inequality of (28) lead to

$$
\int_{\Omega}\left(w^{+}\right)^{2}(t, x) d x \leq C_{2} \int_{0}^{t} \int_{\Omega}\left(\left(w^{+}\right)^{2}+\left(p^{+}\right)^{2}+\left(u^{+}\right)^{2}\right)(\tau, x) d x d \tau
$$

with $C_{2}$ a constant depending on $L, C_{\max }, H^{+}$and $K^{+}$. For the third inequality of (28), we combine similar arguments with

$$
\begin{aligned}
\int_{\Omega} \int_{\Omega} \Phi(x, y) p(\tau, y) p^{+}(\tau, x) d x d y & \leq \Phi_{\max } \int_{\Omega} \int_{\Omega} p^{+}(\tau, y) p^{+}(\tau, x) d x d y \\
& \leq \Phi_{\max }\left(\int_{\Omega} p^{+}(\tau, x) d x\right)^{2} \leq \Phi_{\max }|\Omega| \int_{\Omega}\left(p^{+}\right)^{2}(\tau, x) d x
\end{aligned}
$$


to derive

$$
\int_{\Omega}\left(p^{+}\right)^{2}(t, x) d x \leq C_{3} \int_{0}^{t} \int_{\Omega}\left(\left(p^{+}\right)^{2}+\left(w^{+}\right)^{2}\right)(\tau, x) d x d \tau,
$$

with $C_{3}$ a constant depending on $L, C_{\max }, K^{+}, M^{+}, \Phi_{\max }$ and $|\Omega|$. Adding (29), (30) and (31), and using the Gronwall lemma we get that

$$
\int_{\Omega}\left(\left(u^{+}\right)^{2}+\left(w^{+}\right)^{2}+\left(p^{+}\right)^{2}\right)(t, x) d x=0, \quad \text { for all } 0 \leq t \leq T .
$$

Hence, $u^{+}=w^{+}=p^{+} \equiv 0$ on $\overline{Q_{T}}$, and the comparison principle is proved.

\section{Identification of various regimes}

In this section, we formally identify various behaviors for problem (6) depending on the value of the parameters. Recall that $\beta$ was defined in (16). Here, aridity classes are introduced according to the aridity index which is defined by the ratio of annual rainfall to potential evapotranspiration rate [10]. The aridity is classified into four levels: hyper-arid where bare soil is only stable, arid where bare soil or vegetation patterns are stable, semi-arid where vegetation patterns are only stable and dry-subhumid where uniform vegetation or vegetation patterns are stable.

Hyper-arid ecosystems: $\beta<0$. As seen in Remark 2.4, $\beta<0$ leads to an exponentially fast extinction of plants. In the sequel we exclude this situation and assume $\beta>0$.

Let us investigate the steady states of problem (6), namely nonnegative solutions of

$$
\begin{cases}-\Delta u=r-g H(p) u & x \in \Omega, \\ -d \Delta w+e_{0} w=H(p) u-K(w) p & x \in \Omega, \\ b p=a K(w) p+M(w) c \int_{\Omega} \Phi(x, y) p(t, y) d y & x \in \Omega, \\ \left(\frac{\partial u}{\partial \nu}(x), \frac{\partial w}{\partial \nu}(x)\right)=(0,0) & x \in \partial \Omega .\end{cases}
$$

When looking after solutions $(u, w, p)$ with constant $p$, the third equation yields $p \equiv 0$ or $b=a K(w)+M(w) c$. The former gives $\left(u_{0} \equiv \frac{r}{g H(0)}, w_{0} \equiv \frac{r}{g e_{0}}, p_{0} \equiv 0\right)$. As far as the latter is concerned, since $\beta>0$ and since $K$ and $M$ are increasing,

$$
\exists ! w_{1}>0, a K\left(w_{1}\right)+M\left(w_{1}\right) c=b,
$$

which gives $\left(u_{1} \equiv \frac{r}{g H\left(p_{1}\right)}, w_{1}, p_{1} \equiv \frac{\frac{r}{g}-e_{0} w_{1}}{K\left(w_{1}\right)}\right)$, whose nonnegativity is to be discussed.

Arid ecosystems: $\beta>0, \frac{r}{g}<e_{0} w_{1}$. In this context, $\left(u_{0}, w_{0}, p_{0}\right)$ is the only nonnegative steady state with constant $p$. To study its linear stability, let us consider the associated eigenvalue problem $\left(\operatorname{plug}(u, w, p)(t, x)=\left(u_{0}, w_{0}, p_{0} \equiv 0\right)+\varepsilon e^{-\lambda_{0} t}(\varphi, \psi, \theta)(x)\right.$ into the parabolic problem and keep the $\varepsilon$ terms)

$$
\begin{cases}-\lambda_{0} \varphi-\Delta \varphi=-g H(0) \varphi-r \frac{H^{\prime}(0)}{H(0)} \theta & x \in \Omega, \\ -\lambda_{0} \psi-d \Delta \psi+e_{0} \psi=H(0) \varphi+\frac{r}{g} \frac{H^{\prime}(0)}{H(0)} \theta-K\left(\frac{r}{g e_{0}}\right) \theta & x \in \Omega, \\ -\lambda_{0} \theta+b \theta=a K\left(\frac{r}{g e_{0}}\right) \theta+M\left(\frac{r}{g e_{0}}\right) c \int_{\Omega} \Phi(x, y) \theta(y) d y & x \in \Omega, \\ \left(\frac{\partial \varphi}{\partial \nu}(x), \frac{\partial \psi}{\partial \nu}(x)\right)=(0,0) & x \in \partial \Omega .\end{cases}
$$


Integrating the third equation over $x \in \Omega$, using Fubini-Tonelli theorem and (4) gives

$$
\lambda_{0}=b-a K\left(\frac{r}{g e_{0}}\right)-M\left(\frac{r}{g e_{0}}\right) c .
$$

Since $\frac{r}{g e_{0}}<w_{1}$, we have $\lambda_{0}>b-a K\left(w_{1}\right)-M\left(w_{1}\right) c=0$, so that $\left(u_{0}, w_{0}, p_{0} \equiv 0\right)$ is linearly stable. As far as the eigenfunction is concerned, notice that plugging (35) yields

$$
\int_{\Omega} \Phi(x, y)(\theta(y)-\theta(x)) d y=0, \quad \forall x \in \Omega .
$$

Taking a point $x$ where $\theta$ reaches its maximum and using (3), we see that $\theta$ is constant on a small neighborhood of given size of $x$ and then, by repeating, on the whole of $\Omega$.

The above suggests that, in these arid ecosystems, we again have an exponentially fast extinction of plants.

Semi-arid ecosystems: $\beta>0, \frac{r}{g}>e_{0} w_{1}$. In this context, $\left(u_{0}, w_{0}, p_{0}\right)$ and $\left(u_{1}, w_{1}, p_{1}\right)$ are the two nonnegative steady states with constant $p$. In this regime we have $\lambda_{0}<0$ so that $\left(u_{0}, w_{0}, p_{0}\right)$ is linearly unstable. We now investigate the linear stability of $\left(u_{1}, w_{1}, p_{1}\right)$ by looking at the eigenvalue problem

$$
\begin{cases}-\lambda_{1} \varphi-\Delta \varphi=-g H\left(p_{1}\right) \varphi-r \frac{H^{\prime}\left(p_{1}\right)}{H\left(p_{1}\right)} \theta & x \in \Omega, \\ -\lambda_{1} \psi-d \Delta \psi+e_{0} \psi=H\left(p_{1}\right) \varphi+\frac{r}{g} \frac{H^{\prime}\left(p_{1}\right)}{H\left(p_{1}\right)} \theta-K\left(w_{1}\right) \theta-p_{1} K^{\prime}\left(w_{1}\right) \psi & x \in \Omega, \\ -\lambda_{1} \theta+b \theta=a K\left(w_{1}\right) \theta+a K^{\prime}\left(w_{1}\right) p_{1} \psi & \\ +M\left(w_{1}\right) c \int_{\Omega} \Phi(x, y) \theta(y) d y+M^{\prime}\left(w_{1}\right) c p_{1} \psi & x \in \Omega, \\ \left(\frac{\partial \varphi}{\partial \nu}(x), \frac{\partial \psi}{\partial \nu}(x)\right)=(0,0) & x \in \partial \Omega .\end{cases}
$$

Integrating the third equation over $x \in \Omega$, using Fubini-Tonelli theorem and (33) yields

$$
-\lambda_{1} \int_{\Omega} \theta=\left(a K^{\prime}\left(w_{1}\right)+M^{\prime}\left(w_{1}\right) c\right) p_{1} \int_{\Omega} \psi
$$

so that $\lambda_{1}<0$ (since principal eigenfunctions are positive), so that $p_{1}$ is linearly unstable.

The above suggests that, in these semi-arid ecosystems, there exists in between $p_{0} \equiv 0$ and $p_{1}>0$ a nonconstant steady state $p^{*}(x)$ (possibly spots, stripes...). This will be numerically investigated in subsection 4.2 .

\section{Steady states}

In Section 2, we proved the existence and uniqueness of a global solution to problem (6). Section 3 gave formal discussion on behaviors for problem (6) in some parameter regimes. Then, our subsequent question arises as follows: what kind of solutions do the system (2) exhibit from pattern formation point of view? Since we are interested in vegetation patterns arising in the system, we treat the stationary problem for (2) in this section. 


\subsection{Constant steady states}

We first consider constant equilibrium solutions in the following ODE system associated with (2) (the mean field model):

$$
\begin{aligned}
\frac{d P}{d t} & =a \frac{W}{W+1} P-b P+c \frac{W}{W+1} P, \\
\frac{d W}{d t} & =U \frac{P+w_{U}}{P+1}-\frac{W}{W+1} P-e_{0} W, \\
\frac{d U}{d t} & =r-g U \frac{P+w_{U}}{P+1} .
\end{aligned}
$$

Equilibrium points of (37) are computed as

$$
\left(P_{0}, W_{0}, U_{0}\right):=\left(0, \frac{r}{e_{0} g}, \frac{r}{w_{U} g}\right)
$$

and

$$
\left(P_{1}, W_{1}, U_{1}\right):=\left(\left(\frac{r}{g}-e_{0} W_{1}\right) \frac{W_{1}+1}{W_{1}}, \frac{b}{a+c-b}, \frac{r\left(P_{1}+1\right)}{g\left(P_{1}+w_{U}\right)}\right) .
$$

Since negative equilibria are ecologically meaningless, we focus on nonnegative ones. The equilibrium point $\left(P_{0}, W_{0}, U_{0}\right)$ which indicates desert (bare) states always exists, on the other hand, the existence of the other equilibrium point $\left(P_{1}, W_{1}, U_{1}\right)$ depends on the parameter values, which means that the equilibrium point $\left(P_{1}, W_{1}, U_{1}\right)$ can be negative according to the parameter setting. For example, when the value of $r$ is small, the plant density $P_{1}$ becomes negative. If $P_{1}>0$, then $\left(P_{1}, W_{1}, U_{1}\right)$ indicates vegetation states.

We are interested in the transition of vegetation states when the rainfall rate $r$ varies, because it is one of the important parameters to discuss the possibility of desertification in (2). So, we set $r$ as a bifurcation parameter. Figure 3 shows bifurcation diagrams of equilibrium points for (37) when the rainfall $r$ globally varies. One can see from Figure 3 that $\left(P_{0}, W_{0}, U_{0}\right)=\left(0, \frac{r}{e_{0} g}, \frac{r}{w_{U} g}\right)$, which coincides with bare soil states, is stable for small $r$, but when the value of $r$ increases, it is destabilized at $r=r_{c}=16.9106$ and another branch which corresponds to $\left(P_{1}, W_{1}, U_{1}\right)=\left(\left(\frac{r}{g}-e_{0} W_{1}\right) \frac{W_{1}+1}{W_{1}}, \frac{b}{a+c-b}, \frac{r\left(P_{1}+1\right)}{g\left(P_{1}+w_{U}\right)}\right)$ appears as a consequence of a transcritical bifurcation. The positive part of the branch in Figure 3 (a) corresponds to a vegetated state and it grows linearly as the rainfall rate $r$ increases.

Our original interest is the occurrence of spatial vegetation patterns when the spatial terms are included in the model. Therefore, in the next subsection, we investigate the stability of the positive equilibrium solution for (2) in one space dimension from the view point of the linear stability analysis, and discuss a transition of spatial patterns, depending on a change of a bifurcation parameter: $r$ (rainfall), or $\eta$ (seed dispersal range), or $c$ (seed germination rate). 


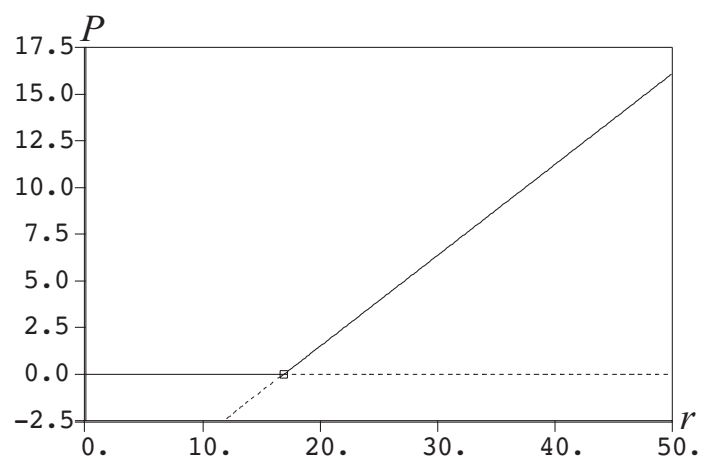

(a) Plant density $P$

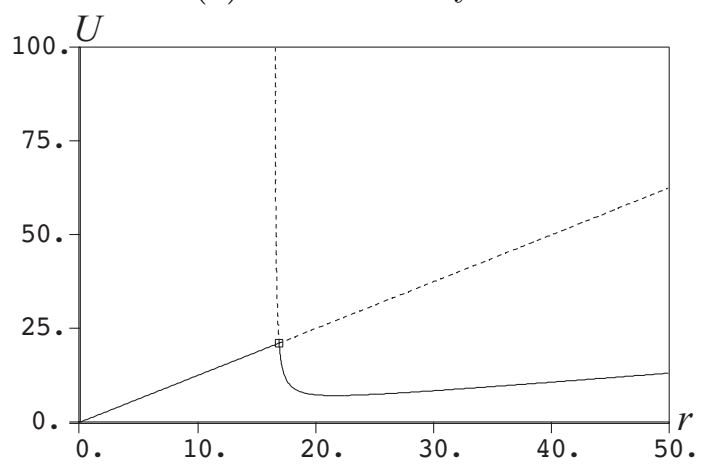

(c) Surface water $U$

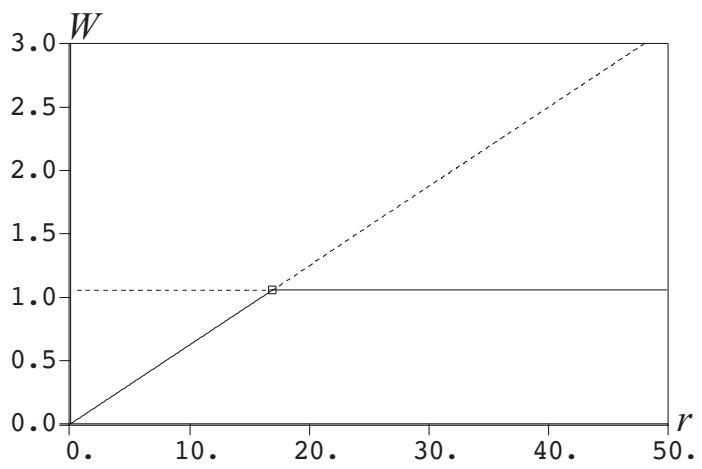

(b) Soil water $W$

Figure 3: Structure of equilibrium points of (37). (a) Plant density $P$. (b) Soil water $W$. (c) Surface water $U$. The horizontal and vertical axes mean the parameter $r$ and the value of the component, respectively. The solid and dashed curves in the figures mean respectively stable and unstable branches. The mark $\square$ in the figures denotes a bifurcation point. The parameter values are $a=10, b=5.2, c=0.12, e_{0}=4, g=4$ and $w_{U}=0.2$. 


\subsection{Global structure of stationary solutions}

In this subsection, we consider the stationary problem in one space dimension

$$
\begin{array}{rlr}
0 & =a \frac{W}{W+1} P-b P+c \frac{W}{W+1} \int_{0}^{L} P(y) \Phi_{\text {approx }}(x, y) d y, & \\
0 & =d \Delta W+U \frac{P+w_{U}}{P+1}-\frac{W}{W+1} P-e_{0} W, & 0<x<L, \\
0 & =\Delta U+r-g U \frac{P+w_{U}}{P+1}, &
\end{array}
$$

under the Neumann boundary conditions $W_{x}(0)=W_{x}(L)=U_{x}(0)=U_{x}(L)=0$. Here, we use the redistribution kernel $\Phi_{\text {approx }}(x, y)$ associated with (5) as shown in Figure 2 . We expect that heterogeneous vegetation patterns emerge as a consequence of bifurcation from the equilibrium solution $\left(P_{1}, W_{1}, U_{1}\right)=\left(\left(\frac{r}{g}-e_{0} W_{1}\right) \frac{W_{1}+1}{W_{1}}, \frac{b}{a+c-b}, \frac{r\left(P_{1}+1\right)}{g\left(P_{1}+w_{U}\right)}\right)$. In order to check this, we first carry out the linear stability analysis around the equilibrium solution. The system (2) is linearized around the equilibrium solution $\left(P_{1}, W_{1}, U_{1}\right)$ as follows:

$$
\left(\begin{array}{c}
\tilde{P}_{t} \\
\tilde{W}_{t} \\
\tilde{U}_{t}
\end{array}\right)=\left(\begin{array}{ccc}
c \frac{W_{1}}{W_{1}+1} T+a \frac{W_{1}}{W_{1}+1}-b & (a+c) \frac{P_{1}}{\left(W_{1}+1\right)^{2}} & 0 \\
U_{1} \frac{1-w_{U}}{\left(P_{1}+1\right)^{2}}-\frac{W_{1}}{W_{1}+1} & d \frac{\partial^{2}}{\partial x^{2}}-\frac{P_{1}}{\left(W_{1}+1\right)^{2}}-e_{0} & \frac{P_{1}+w_{U}}{P_{1}+1} \\
-g U_{1} \frac{1-w_{U}}{\left(P_{1}+1\right)^{2}} & 0 & \frac{\partial^{2}}{\partial x^{2}}-g \frac{P_{1}+w_{U}}{P_{1}+1}
\end{array}\right)\left(\begin{array}{c}
\tilde{P} \\
\tilde{W} \\
\tilde{U}
\end{array}\right),
$$

where $T$ is an operator defined by $(T \tilde{P})(x)=\int_{0}^{L} \tilde{P}(y) \Phi_{\text {approx }}(x, y) d y$. For the linear stability analysis, it is necessary to know a series of eigenvalues for the operator $T$. However, they are not obtained by hand, so we numerically compute the eigenvalues $\rho_{n}(n=1,2, \cdots)$ such that $\int_{0}^{L} \cos \left(\frac{n \pi y}{L}\right) \Phi_{\text {approx }}(x, y) d y=\rho_{n} \cos \left(\frac{n \pi x}{L}\right)$. When we set $\xi=0.088587, \eta=400$ and $L=0.25$, the eigenvalues of the operator $T$ are obtained as follows:

$$
\begin{array}{llll}
\rho_{1}=0.906018, & \rho_{2}=0.673825, & \rho_{3}=0.411369, & \rho_{4}=0.206153, \\
\rho_{5}=0.084805, & \rho_{6}=0.028637, & \rho_{7}=0.007938, & \rho_{8}=0.001806, \\
\rho_{9}=0.000337, & \rho_{10}=0.000052, & \ldots . &
\end{array}
$$

Therefore, for each Fourier mode, we have a series of linear systems of ordinary differential equations

$$
\left(\begin{array}{c}
\tilde{P}_{n t} \\
\tilde{W}_{n t} \\
\tilde{U}_{n t}
\end{array}\right)=\left(\begin{array}{ccc}
c \frac{W_{1}}{W_{1}+1} \rho_{n}+a \frac{W_{1}}{W_{1}+1}-b & (a+c) \frac{P_{1}}{\left(W_{1}+1\right)^{2}} & 0 \\
U_{1} \frac{1-w_{U}}{\left(P_{1}+1\right)^{2}}-\frac{W_{1}}{W_{1}+1} & -d\left(\frac{n \pi}{L}\right)^{2}-\frac{P_{1}}{\left(W_{1}+1\right)^{2}}-e_{0} & \frac{P_{1}+w_{U}}{P_{1}+1} \\
-g U_{1} \frac{1-w_{U}}{\left(P_{1}+1\right)^{2}} & 0 & -\left(\frac{n \pi}{L}\right)^{2}-g \frac{P_{1}+w_{U}}{P_{1}+1}
\end{array}\right)\left(\begin{array}{c}
\tilde{P}_{n} \\
\tilde{W}_{n} \\
\tilde{U}_{n}
\end{array}\right),
$$

$n=1,2,3, \cdots$. Investigating zero eigenvalues of these linearized matrices, we know the location of bifurcation points on the constant solution branch $\left(P_{1}, W_{1}, U_{1}\right)$. When we focus on the parameters $r$ and $d$, a bifurcation curve for $n$ mode, where an eigenvalue takes zero, is obtained as

$$
\begin{aligned}
\Gamma_{n}= & \left\{(r, d) \in \mathbb{R}^{2} \mid\right. \\
& \left(c \frac{W_{1}}{W_{1}+1} \rho_{n}+a \frac{W_{1}}{W_{1}+1}-b\right)\left(d\left(\frac{n \pi}{L}\right)^{2}+\frac{P_{1}}{\left(W_{1}+1\right)^{2}}+e_{0}\right)\left(\left(\frac{n \pi}{L}\right)^{2}+g \frac{P_{1}+w_{U}}{P_{1}+1}\right) \\
& +\left((a+c) \frac{P_{1}}{\left(W_{1}+1\right)^{2}}\right)\left(\frac{P_{1}+w_{U}}{P_{1}+1}\right)\left(-g U_{1} \frac{1-w_{U}}{\left(P_{1}+1\right)^{2}}\right) \\
& \left.+\left((a+c) \frac{P_{1}}{\left(W_{1}+1\right)^{2}}\right)\left(U_{1} \frac{1-w_{U}}{\left(P_{1}+1\right)^{2}}-\frac{W_{1}}{W_{1}+1}\right)\left(\left(\frac{n \pi}{L}\right)^{2}+g \frac{P_{1}+w_{U}}{P_{1}+1}\right)=0\right\} .
\end{aligned}
$$


Based on the bifurcation curves, we know the stable and unstable regions of the equilibrium solution $\left(P_{1}, W_{1}, U_{1}\right)$ in the $(r, d)$-plane. Figure 4 indicates the bifurcation curves $\left\{\Gamma_{n}\right\}$ and

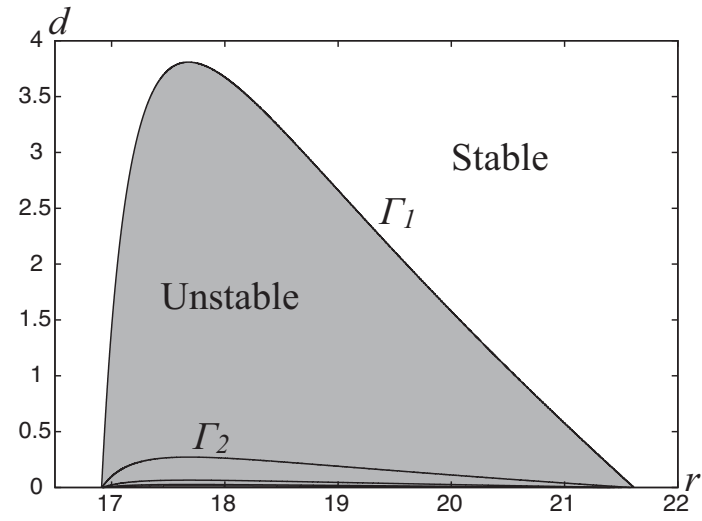

Figure 4: Bifurcation curves, and stable and unstable regions of the constant solution $\left(P_{1}, W_{1}, U_{1}\right)$ for $(38)$ with (5). The horizontal and vertical axes mean the parameters $r$ and $d$, respectively. The other parameter values are the same as the ones in Figures 2 and 3.

the stable and unstable regions for the equilibrium solution. We can see from the figure that for a suitably fixed value of $d$, the stability of the equilibrium solution $\left(P_{1}, W_{1}, U_{1}\right)$ changes from stable state into unstable one as the value $r$ increases from $r=r_{c}$. When the value $r$ increases further, it recovers the stability. Moreover, we can expect that the one-mode solution primarily bifurcates from the constant solution branch in this parameter setting when the value of $r$ increases.

Based on Figure 4, we numerically compute a global bifurcation diagram by using a numerical bifurcation software AUTO [5]. Figure 5 shows a bifurcation diagram for $d=0.1$ when the bifurcation parameter $r$ globally varies. For small values of $r$, the bare state $\left(P_{0}, W_{0}, U_{0}\right)=\left(0, \frac{r}{e_{0} g}, \frac{r}{w_{U} g}\right)$ is stable. As the value $r$ increases, the homogeneous vegetation state $\left(P_{1}, W_{1}, U_{1}\right)=\left(\left(\frac{r}{g}-e_{0} W_{1}\right) \frac{W_{1}+1}{W_{1}}, \frac{b}{a+c-b}, \frac{r\left(P_{1}+1\right)}{g\left(P_{1}+w_{U}\right)}\right)$ appears stably due to the transcritical bifurcation at $r=r_{c}=16.9106$ while the bare state $\left(0, \frac{r}{e_{0} g}, \frac{r}{w_{U} g}\right)$ becomes unstable. However, this uniform vegetated branch undergoes a subcritical pitchfork bifurcation at $r=16.9167$ and becomes unstable (see the enlarged figure in Figure 5). Although the nonconstant branches resulting from the bifurcation point are unstable, they become stable via a saddle-node bifurcation at $r=15.5772$ and keeps the stability up to $r=25.5270$. And the saddle-node bifurcations at $r=25.5270$ change the stability of the branches and they connect to the constant solution branch which means uniform vegetation states at $r=21.5037$ as a subcritical pitchfork bifurcation. Here, we note that a pair of nonconstant stationary solution branches appears as a result of a pitchfork bifurcation, that is the upper and lower branches in Figure 5. Actually, we can identify these two branches because the stationary solution on the lower branch corresponds to $(P(L-x), W(L-x), U(L-x))$ if a triplet $(P(x), W(x), U(x))$ is a stationary solution on the upper branch. Therefore, the stability of these two branches coincides. Stable nonconstant stationary solutions observed in the range $15.5772<r<25.5270$ correspond to nonconstant stable vegetation patterns. In addition, one can see from the figure that there exist bistable regions in $15.5772<r<16.9167$ and $21.5037<r<25.5270$. That is, there are two stable stationary solutions, a uniform state and a heterogeneous state 


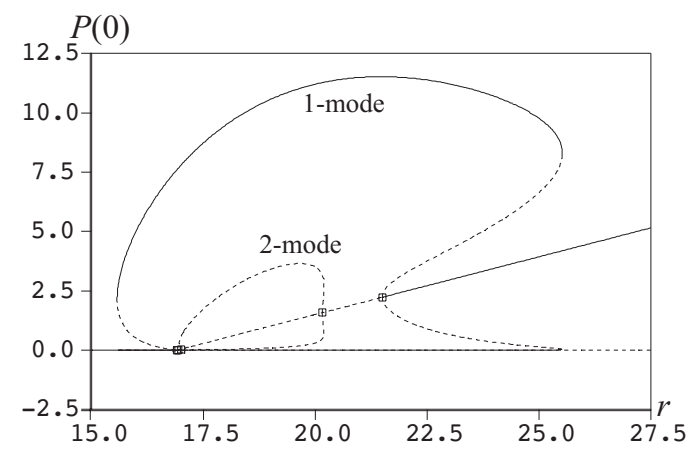

Global bifurcation diagram

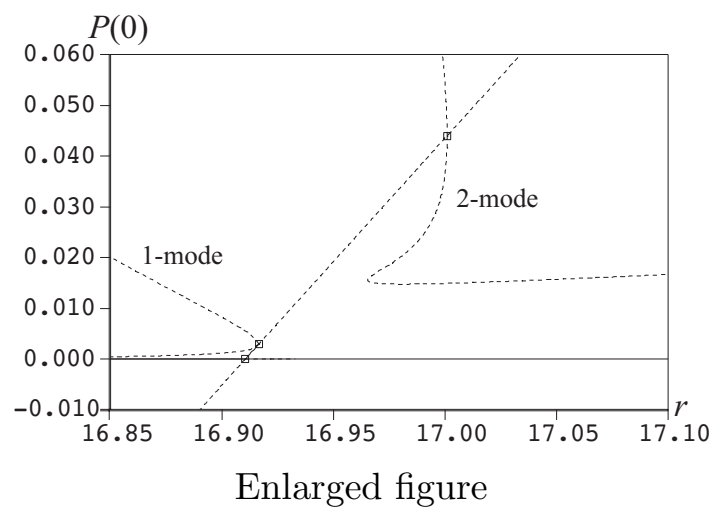

Figure 5: Structure of steady states of (38) with (5). The horizontal and vertical axes mean the parameter $r$ and the value of $P$ at $x=0$. The solid and dashed curves in the figures mean respectively stable and unstable branches. The mark $\square$ in the figure denote a bifurcation point. Each of the branches corresponds to 1-Fourier mode and 2-Fourier mode. The parameter values are $a=10, b=5.2, c=0.12, d=0.1, e_{0}=4, g=4, w_{U}=0.2, \eta=400$ and $L=0.25$.

in these parameter regions. The profiles of stable stationary solutions for some values of $r$ are shown in Figure 6. We can observe that the amounts of average biomass $\frac{1}{L} \int_{0}^{L} P(x) d x$ increase as the rainfall parameter $r$ increases. When the vertical axis $P(0)$ in Figure 5 is replaced by the average biomass $\frac{1}{L} \int_{0}^{L} P(x) d x$, the global bifurcation diagram is shown in Figure 7. This figure indicates that the biomass of heterogeneous steady states is always higher than that of constant steady states for each value of $r$. Therefore, aggregation formation is more reasonable for plants in drylands than uniform vegetation from the viewpoint of a survival strategy.

Seed dispersal range as the bifurcation parameter. Next, we change the bifurcation parameter from $r$ to $\eta$ in (5) to investigate how the seed dispersal range influences vegetation patterns. Here, we note that small values of $\eta$ in (5) correspond to long-range seed dispersal and large ones correspond to short-range seed dispersal. Figure 8 shows a global structure of steady states of (38) with (5) when the parameter $\eta$ globally varies. When the value of $\eta$ is small, the homogeneous steady state $\left(P_{1}, W_{1}, U_{1}\right)$ only is stable. This means that the long-range seed dispersal tends to stabilize uniform vegetated states. As the value of $\eta$ increases, the homogeneous steady state is destabilized at $\eta=67.4238$ due to a subcritical pitchfork bifurcation and a pair of branches of nonconstant steady state appears. However, these branches become stable because of the saddle-node bifurcations at $\eta=39.9782$. As a result, stable nonconstant vegetated states are observed for $\eta>39.9782$. In addition, we know that another stable vegetated states appear from $\eta=1239.08$. Figure 9 displays some profiles of stable steady states in Figure 8. Since there are two stable branches for the large $\eta$ value, the two stable steady states for $\eta=1400$ are shown in Figure 9 . These results imply that short-range seed dispersal (the large values of $\eta$ ) raises the maximum value of $P$ and promotes multiple stable patterns.

Seed germination rate as the bifurcation parameter. Last, we investigate the influence of the seed germination rate $c$ on vegetation patterns. So, we set the parameter $c$ as a bifurcation parameter. When the value of $c$ is large, that is, the germination rate is high, we 


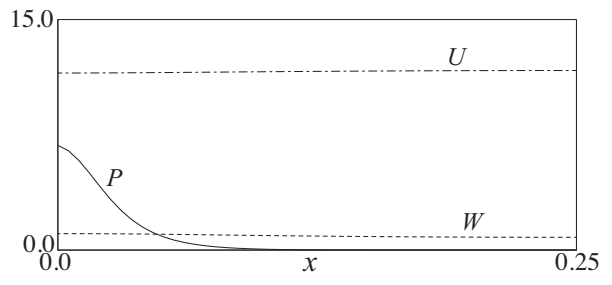

$r=16.6$

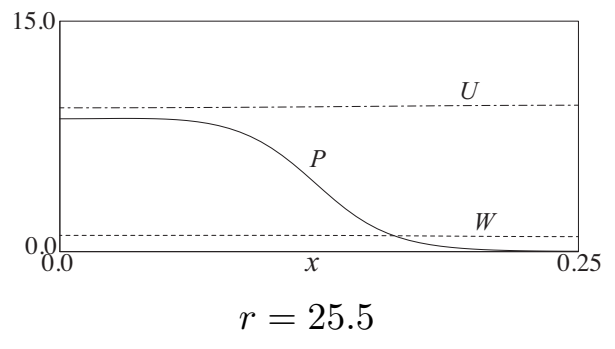

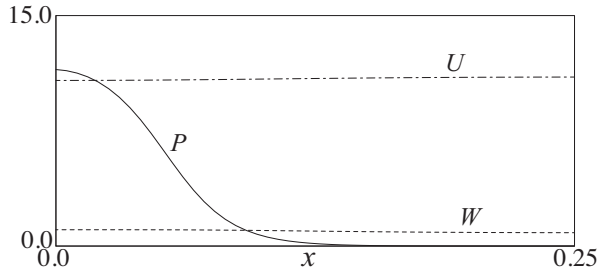

$r=21$

Figure 6: Profiles of stable stationary solutions in (38) for some values $r$. The solid, dashed and long dashed short dashed curves indicate $P, W$ and $U$, respectively. The other parameter values are the same as the ones in Figure 5.

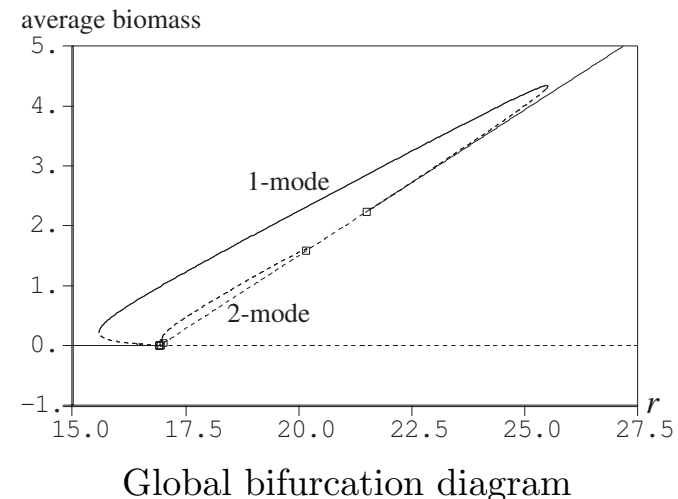

Global bifurcation diagram

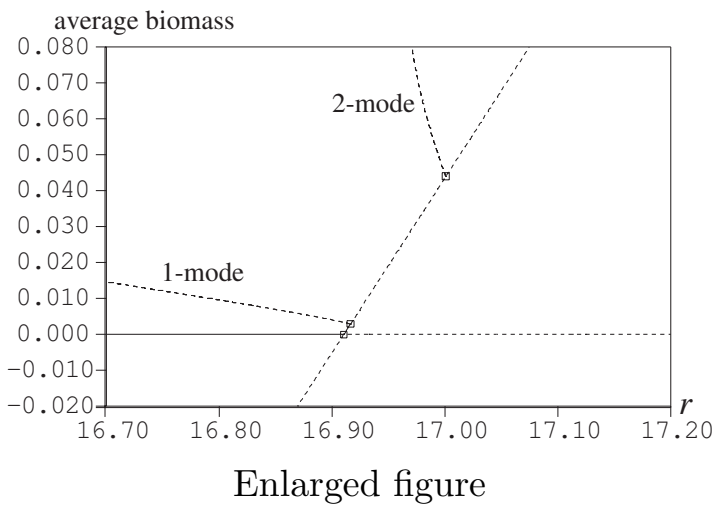

Figure 7: Structure of steady states of (38) with (5). The horizontal and vertical axes mean the parameter $r$ and the average biomass $\frac{1}{L} \int_{0}^{L} P(x) d x$. The parameter values are the same as the ones in Figure 5. 


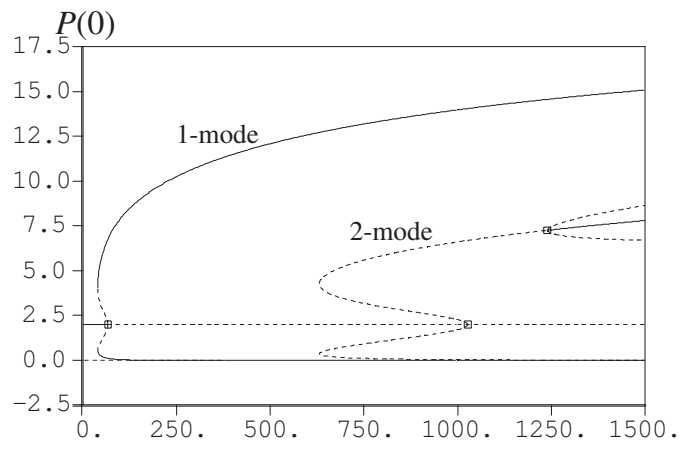

(a)

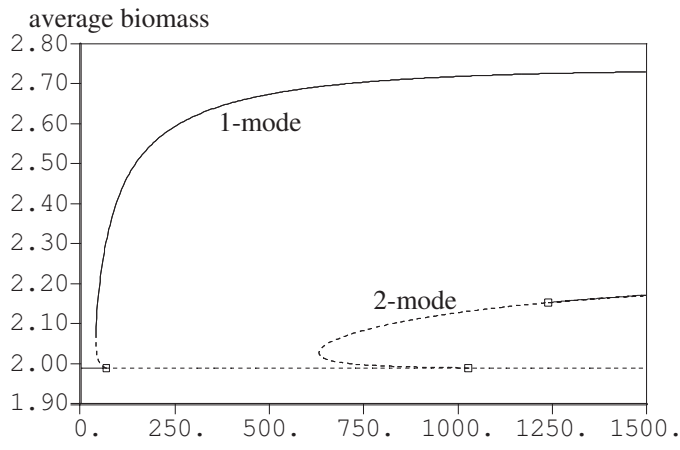

(b)

Figure 8: Structure of steady states of (38) with (5). Each of the branches corresponds to 1-Fourier mode and 2-Fourier mode. The parameter values are $a=10, b=5.2, c=0.12$, $d=0.1, e_{0}=4, g=4, w_{U}=0.2, r=21$ and $L=0.25$. (a) The horizontal and vertical axes mean the parameter $\eta$ and the value of $P$ at $x=0$. (b) The horizontal and vertical axes mean the parameter $\eta$ and the average biomass $\frac{1}{L} \int_{0}^{L} P(x) d x$.
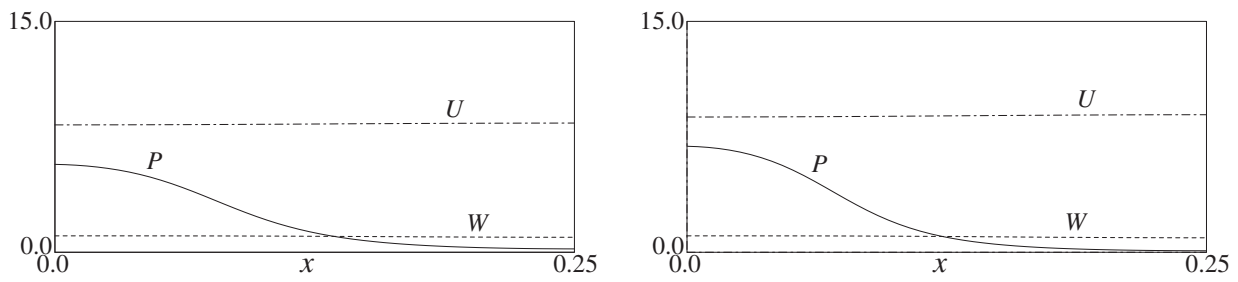

$\eta=50$

$\eta=70$
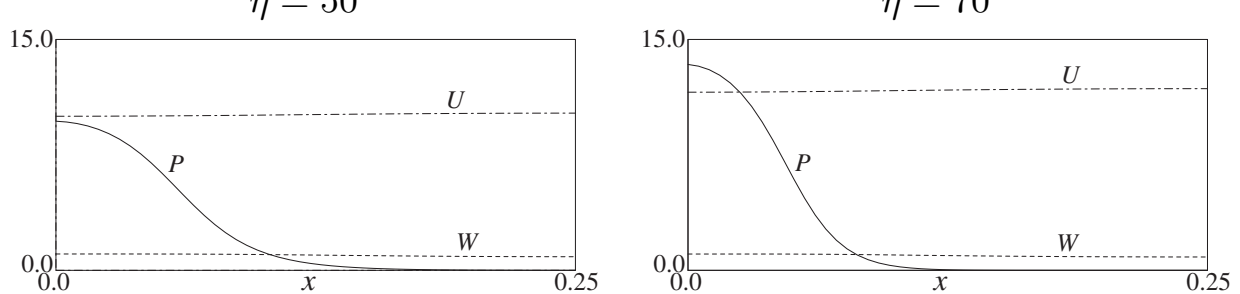

$\eta=200$

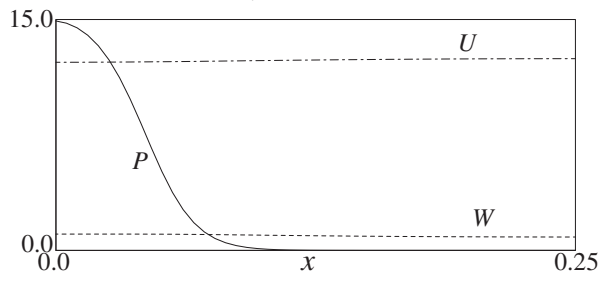

$\eta=800$

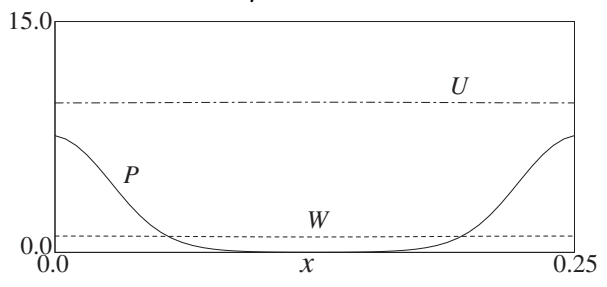

$\eta=1400$

$\eta=1400$

Figure 9: Profiles of stable stationary solutions in (38) for some values $\eta$. The solid, dashed and long dashed short dashed curves indicate $P, W$ and $U$, respectively. The other parameter values are the same as the ones in Figure 8. 
can expect that the homogeneous vegetated states is stable. Actually, as shown in Figure 10

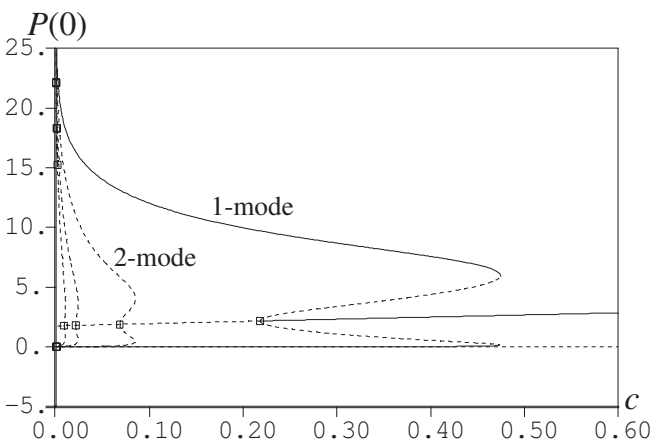

(a)

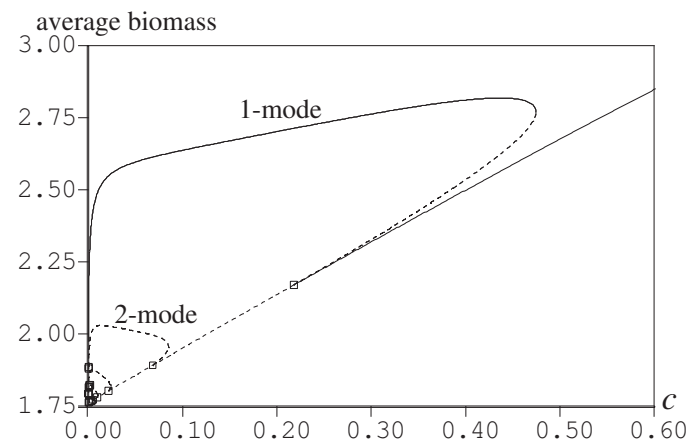

(b)

Figure 10: Structure of steady states of (38) with (5). Each of the branches corresponds to 1-Fourier mode, 2-Fourier mode, 3-Fourier mode and 4-Fourier mode. The parameter values are $a=10, b=5.2, d=0.1, e_{0}=4, g=4, w_{U}=0.2, r=21, \eta=400$ and $L=0.25$. (a) The horizontal and vertical axes mean the parameter $c$ and the value of $P$ at $x=0$. (b) The horizontal and vertical axes mean the parameter $c$ and the average biomass $\frac{1}{L} \int_{0}^{L} P(x) d x$.

which indicates a global bifurcation diagram when the germination rate $c$ varies, one can see that the homogeneous steady state is stable for large $c$ values. As the value of $c$ decreases, it is destabilized at $c=0.21761983$ via a subcritical pitchfork bifurcation and branches of nonconstant steady states emerge, but the saddle-node bifurcations at $c=0.47446$ stabilize these branches. So, nonconstant stable vegetated states are observed for $c<0.47446$. Figure 11 indicates the profiles of stable steady states for $c=0.001, c=0.05, c=0.2$ and $c=0.4$. Figures 10 and 11 imply that the maximum value of $P$ increases and the vegetated region gets narrow as the value of $c$ decreases.

\subsection{Global structure of stationary solutions for other kernels}

So far, we have discussed global structures of stationary solutions of (38) together with the kernel (5) in one space dimension. However, we have several choices for the kernel $k(x, y)$. In this subsection, we numerically show global structures of stationary solutions for other types of kernels and discuss the variation of bifurcation diagrams according to the choice of kernels.

We here consider the following three types of kernels:

$$
\begin{gathered}
k(x, y)=\frac{1}{\xi} e^{-\eta|x-y|}, \\
k(x, y)=\frac{1}{\xi} \max \left\{1-\eta|x-y|^{2}, 0\right\}
\end{gathered}
$$

and

$$
k(x, y)=\frac{1}{\xi}\left(e^{-\eta(x-y-\zeta)^{2}}+e^{-\eta(x-y+\zeta)^{2}}\right) .
$$

The exponential kernel (40) has a cusp and a tail, whereas the quadratic kernel (41) has no tail (see Figure 12(a)). On the other hand, the kernel (42) possesses a bimodal shape for suitable values of $\zeta$. Figure 12(b) displays kernel shapes of (42) for some $\zeta$ values. When a 

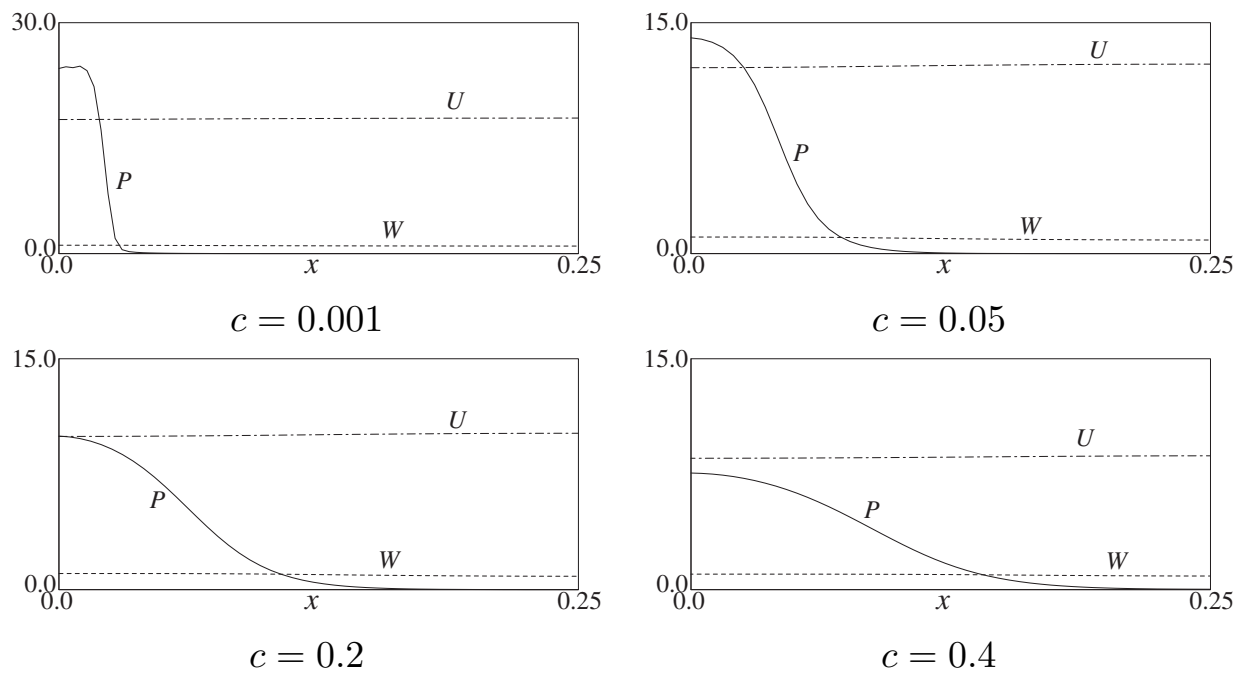

Figure 11: Profiles of stable stationary solutions in (38) for some values $c$. The solid, dashed and long dashed short dashed curves indicate $P, W$ and $U$, respectively. The other parameter values are the same as the ones in Figure 10.

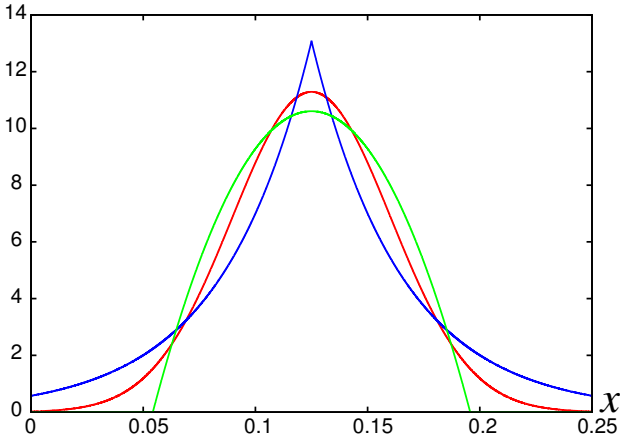

(a)

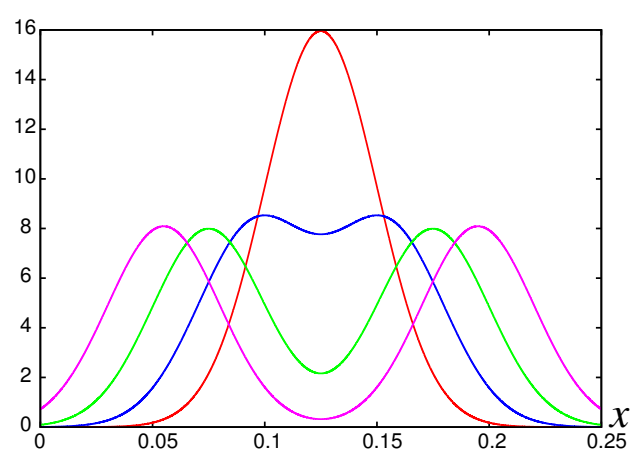

(b)

Figure 12: (a) (red) kernel shape (5) with $\eta=400$. (blue) kernel shape (40) with $\eta=25$. (green) kernel shape (41) with $\eta=200$. The horizontal axis means $x$ and $y=0.125$. (b) kernel shape (42) with $\eta=800$. (red) $\zeta=0$. (blue) $\zeta=0.03$. (green) $\zeta=0.05$. (magenta) $\zeta=0.07$. The horizontal axis means $x$ and $y=0.125$. 
plant disperses the seeds far away, it is supposed that the peak of the seed density is located away from the position of the plant. For describing this situation, bimodal kernel shapes like Figure 12(b) may be suitable.

For unimodal cases, we give global bifurcation diagrams for (38) with (40) and (38) with (41) in Figures 13 and 14, respectively. It turns out that qualitatively similar bifurcation

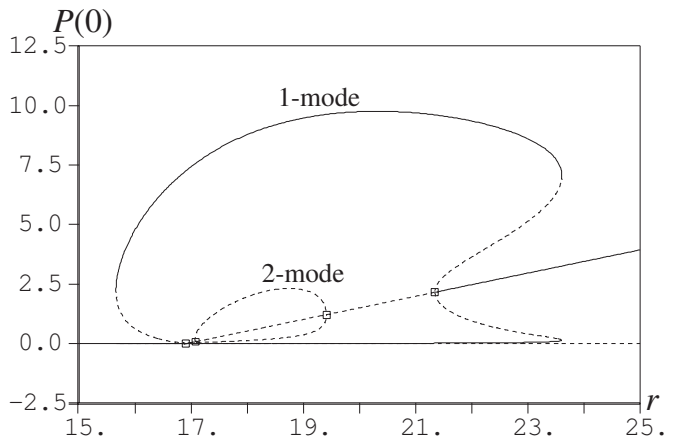

(a)

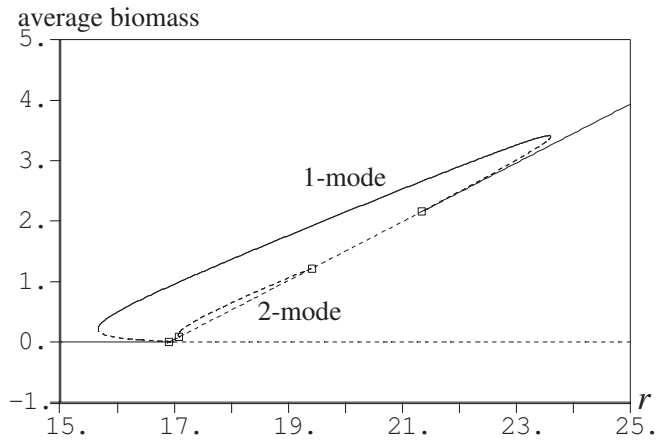

(b)

Figure 13: Structure of steady states of (38) with (40). Each of the branches corresponds to 1 -Fourier mode and 2-Fourier mode. The parameter values are $a=10, b=5.2, c=0.12$, $d=0.1, e_{0}=4, g=4, w_{U}=0.2, \eta=25$ and $L=0.25$. (a) The horizontal and vertical axes are the parameter $r$ and the values of $P$ at $x=0$, respectivey. (b) The horizontal and vertical axes are the parameter $r$ and the average biomass $\frac{1}{L} \int_{0}^{L} P(x) d x$, respectivey.

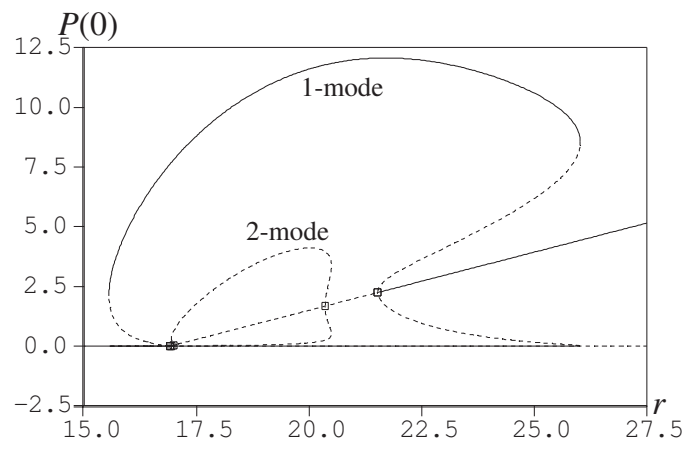

(a)

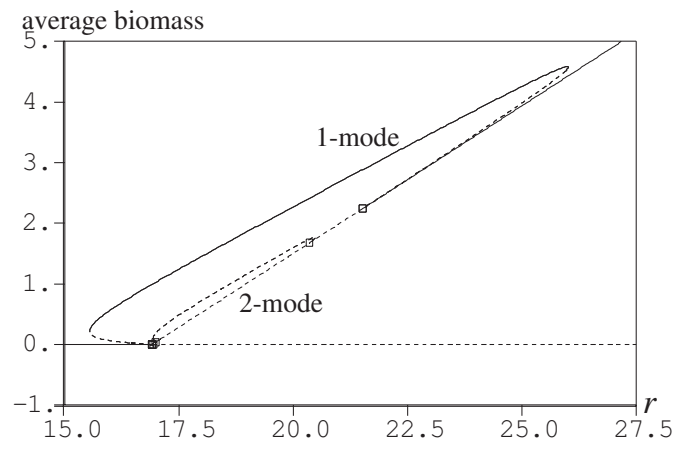

(b)

Figure 14: Structure of steady states of (38) with (41). Each of the branches corresponds to 1-Fourier mode and 2-Fourier mode. The parameter values are $a=10, b=5.2, c=0.12$, $d=0.1, e_{0}=4, g=4, w_{U}=0.2, \eta=200$ and $L=0.25$. (a) The horizontal and vertical axes are the parameter $r$ and the values of $P$ at $x=0$, respectivey. (b) The horizontal and vertical axes are the parameter $r$ and the average biomass $\frac{1}{L} \int_{0}^{L} P(x) d x$, respectivey.

diagrams to Figures 5 and 7 are obtained. When we focus on the unimodal case, these results suggest that the choice of suitable kernels is not of great influence on the global structure of stationary solutions. That is, if the kernel is a unimodal type, a similar tendency of vegetation patterns may be exhibited. 
Next, we display global structures of steady states of (38) with (42) for some $\zeta$ values in Figure 15. When $\zeta=0$, the kernel possesses a unimodal shape as shown in Figure
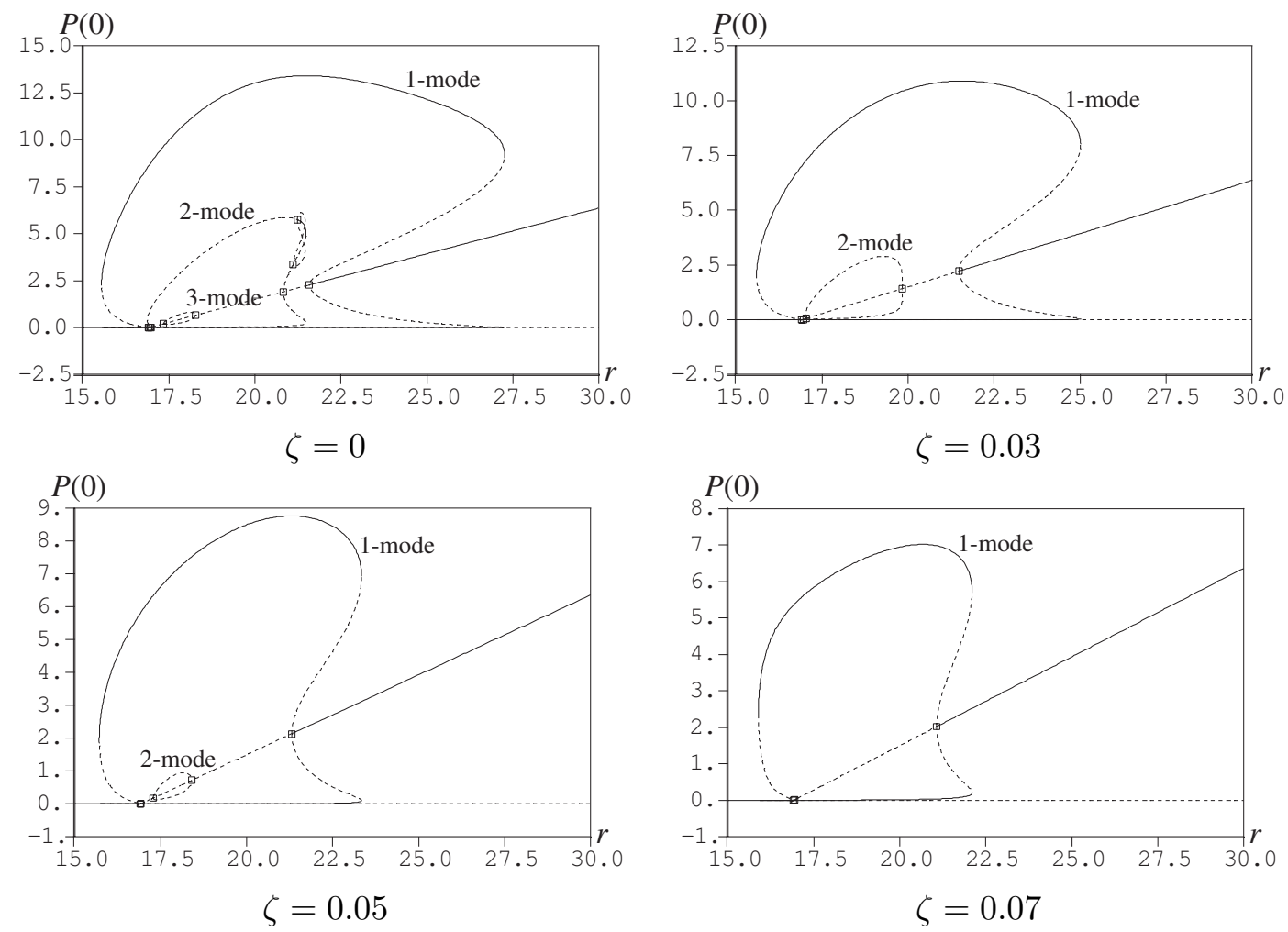

Figure 15: Structure of steady states of (38) with (42). The horizontal and vertical axes mean the parameter $r$ and the values of $P$ at $x=0$, respectivey. Each of the branches corresponds to 1-Fourier mode, 2-Fourier mode and 3-Fourier mode. The parameter values are $a=10$, $b=5.2, c=0.12, d=0.1, e_{0}=4, g=4, w_{U}=0.2, \eta=800$ and $L=0.25$.

12(b). In this case, we observe stable and heterogeneous vegetation states in the range $15.5383<r<27.2551$. Besides, there are bistable regions which mean the coexistence of uniform steady states and heterogeneous ones in $15.5383<r<16.9137$ and $21.5716<$ $r<27.2551$. As the value of $\zeta$ increases, the two peaks of the kernel are located away from the center as shown in Figure 12(b). Then, global structures of steady states when the value $\zeta$ increases vary gradually. In particular, the parameter region where stable and heterogeneous vegetation states are observed becomes narrow: $15.5954<r<25.0128$ for $\zeta=0.03,15.7118<r<23.3372$ for $\zeta=0.05$ and $15.8872<r<22.0956$ for $\zeta=0.07$. Figure 16 shows a global structure of steady states of (38) with (42) when the value $\zeta$ varies as a bifurcation parameter. One can see from the figure that the homogeneous vegetation state $\left(P_{1}, W_{1}, U_{1}\right)$ is stable for large $\zeta$ values, but it is destabilized by a subcritical pitchfork bifurcation at $\zeta=0.075173$ when the value $\zeta$ decreases. And, the saddle-node bifurcations at $\zeta=0.095363$ stabilize the unstable and nonconstant stationary solution branches emerging from $\zeta=0.075173$. Therefore, stable and nonconstant stationary solutions are observed for $\zeta<0.095363$. Figure 17 exhibits profiles of stable stationary solutions for some $\zeta$ values. As the value of $\zeta$ increases, the maximum value of $P$ decreases. These results imply that the 


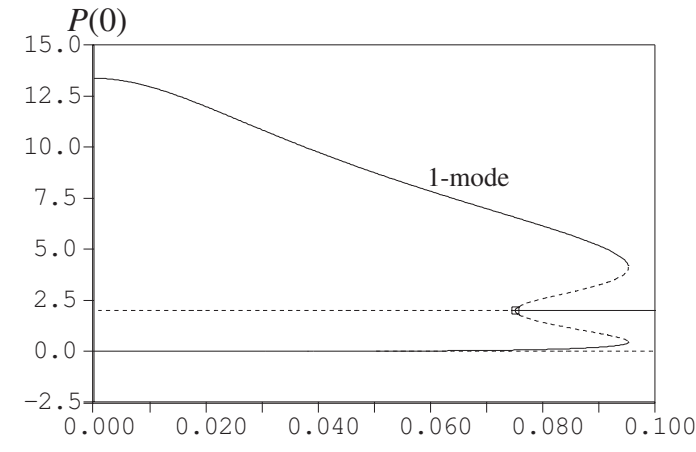

(a)

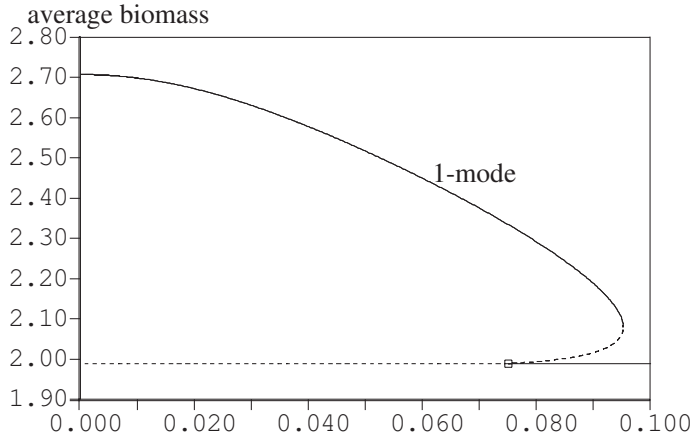

(b)

Figure 16: Structure of steady states of (38) with (42). The branch corresponds to 1-Fourier mode. The parameter values are $a=10, b=5.2, c=0.12, d=0.1, e_{0}=4, g=4, w_{U}=0.2$, $r=21, \eta=800$ and $L=0.25$. (a) The horizontal and vertical axes are the parameter $\zeta$ and the values of $P$ at $x=0$, respectivey. (b) The horizontal and vertical axes are the parameter $\zeta$ and the average biomass $\frac{1}{L} \int_{0}^{L} P(x) d x$, respectivey.
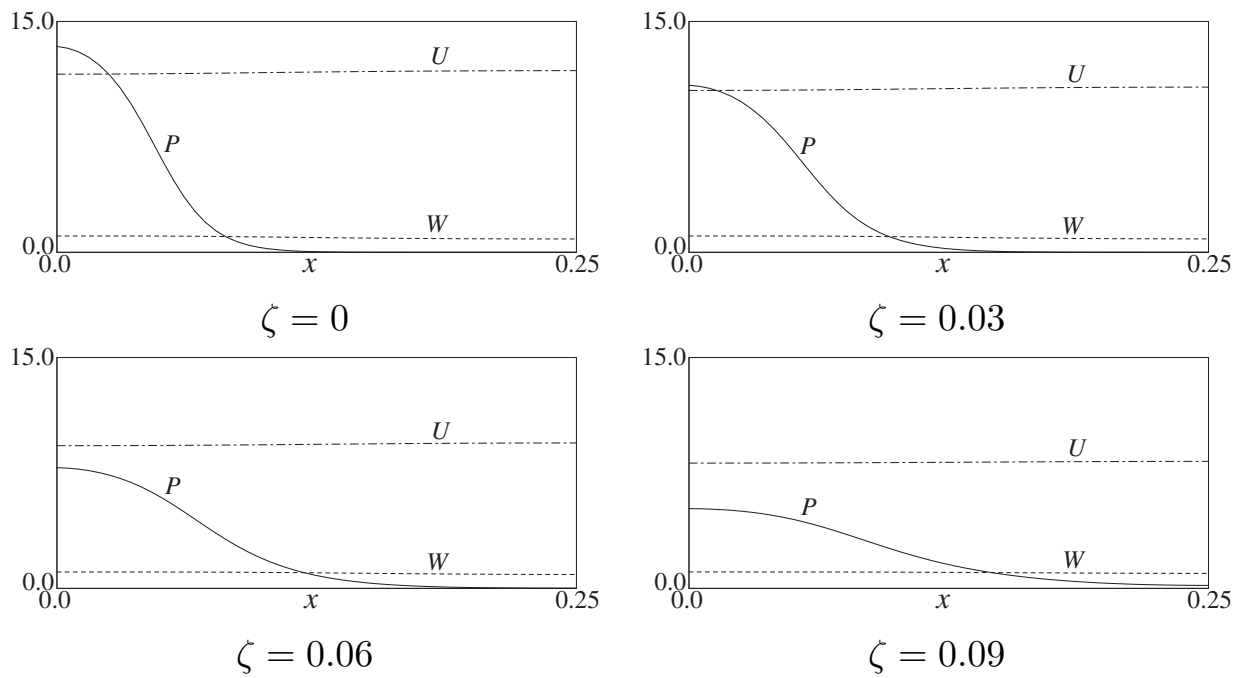

Figure 17: Profiles of stable stationary solutions in (38) with (42) for some parameter values $\zeta$. The solid, dashed and long dashed short dashed curves indicate $P, W$ and $U$, respectively. The other parameter values are the same as the ones in Figure 16. 
kernel (42) with the large value of $\zeta$ tends to inhibit heterogeneity in this system.

\subsection{Two dimensional vegetation patterns}

In the previous subsections, we discussed the one-dimensional problem from the viewpoint of bifurcation diagrams. We here consider the two-dimensional problem and discuss a transition of vegetation patterns in a two dimensional domain when parameter values vary. We focus on four parameters which are taken into account in the previous subsections and investigate relations between vegetation patterns and the variation of the parameter values. The first is the rainfall rate $r$, whose parameter is related to climate change. Figure 18 shows vegetation patterns $P$ at $t=5000$ when the rainfall rate $r$ changes. Here, we compute (2) together
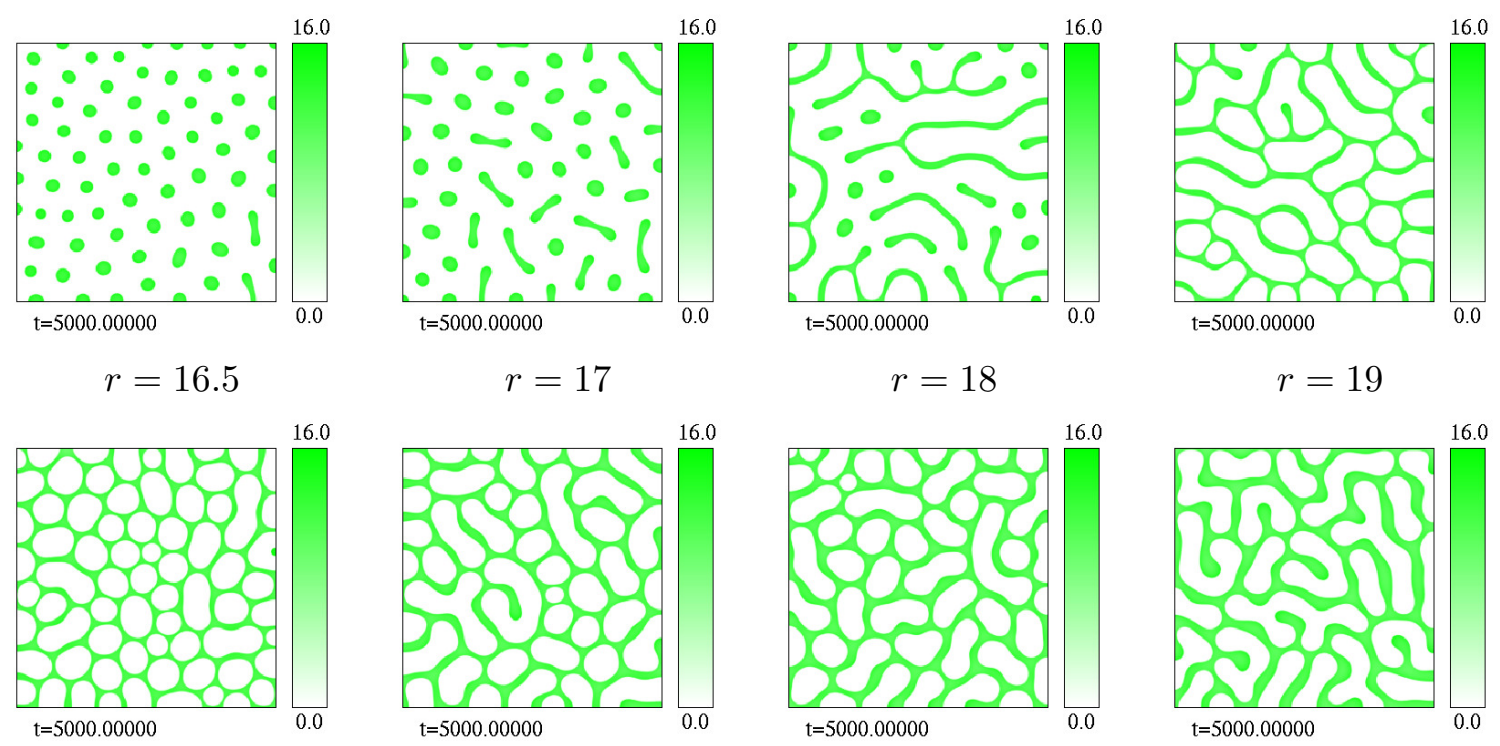

$$
r=20
$$

$$
r=21
$$

$$
r=22
$$

$$
r=23
$$
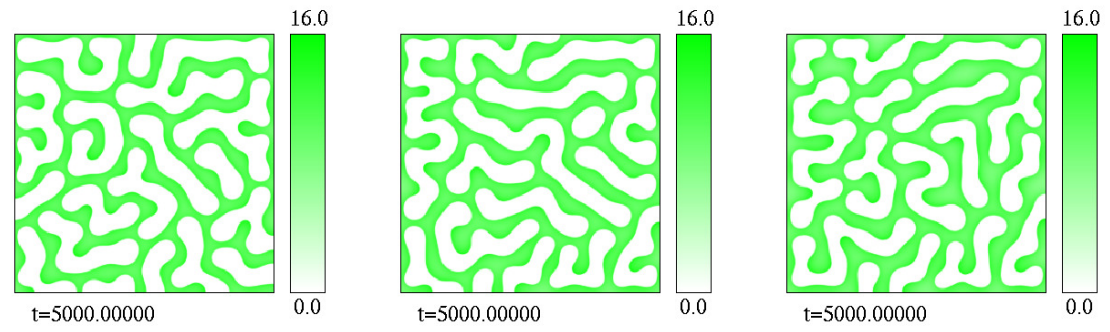

$$
r=24
$$

$r=25$

$r=26$

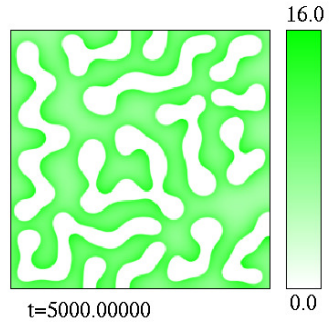

$r=27$

Figure 18: Vegetation patterns $P$ on a square domain $(0,15) \times(0,15)$ when the value of $r$ varies. The other parameter values are the same as the ones in Figure 5. The color scale indicates that green color corresponds to high plant density and white color corresponds to less plant density.

with the two dimensional version of (5) under the Neumann boundary conditions and initial conditions $\left(P_{0}, W_{0}, U_{0}\right)$ or $\left(P_{1}, W_{1}, U_{1}\right)$ with spatially nonuniform perturbations. We have confirmed that patterns at $t=5000$ presented in this paper are almost steady states. For the 
small values of $r$ and for the large values of $r$, we numerically observed no vegetation states $\left(P_{0}, W_{0}, U_{0}\right)$ and uniform vegetation states $\left(P_{1}, W_{1}, U_{1}\right)$, respectively. For the moderate values of $r$, heterogeneous vegetation patterns are exhibited. As the rate $r$ increases, spot patterns $(r=16.5)$, stripe patterns $(r=18,19)$ and gap patterns $(r=20)$ are observed. These results indicate that vegetation patterns change according to the rainfall rate $r$, which is a good agreement with field observations. However, when the value $r$ increases further, the system (2) surprisingly exhibits complicated patterns like a labyrinth which are displayed in Figure $18(r=22-27)$. Depending on the value of $r$, the stripe width of the vegetation patterns gets thicker. For $r>30$, uniform vegetation states are observed. We note that the parameter region where the labyrinth patterns are observed $(22<r<30)$ is bistable, that is, homogeneous vegetation states and heterogeneous ones coexist in this parameter region.

Secondly, we show a transition of two-dimensional vegetation patterns when the parameter $\eta$ varies, which corresponds to Figure 8 in the one-dimensional case. Here, we note that the parameter $\eta$ means the seed dispersal range. When the value of $\eta$ is small, that is longrange seed dispersal, uniform vegetation states are obtained as well as in the one-dimensional case. As the value of $\eta$ is large, namely short-range seed dispersal strategy, heterogeneous vegetation patterns are observed as shown in Figure 19. Depending on the seed dispersal

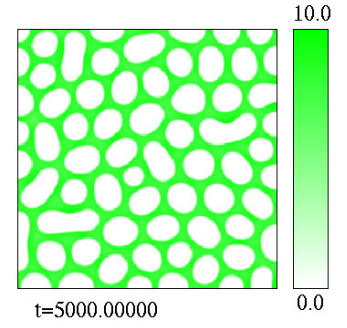

$\eta=50$

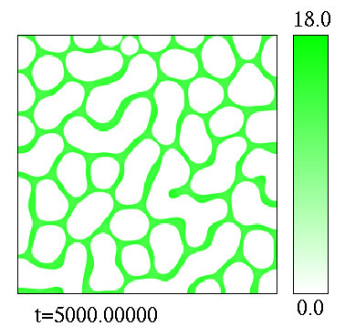

$\eta=1200$

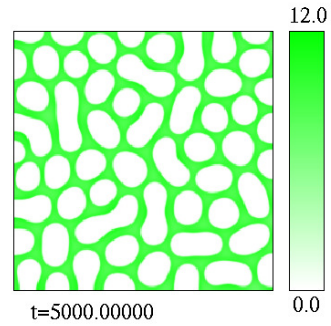

$\eta=100$

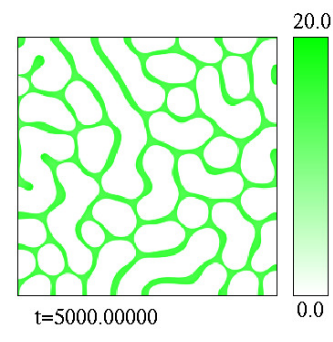

$\eta=2000$

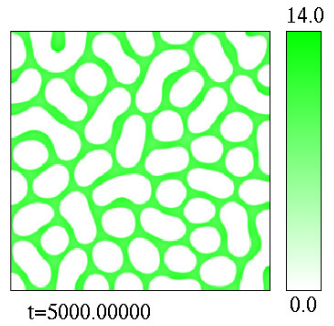

$\eta=200$

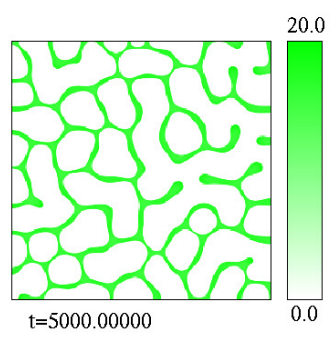

$\eta=4000$

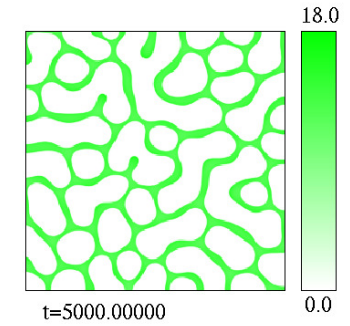

$$
\eta=800
$$

Figure 19: Vegetation patterns $P$ on a square domain $(0,15) \times(0,15)$ when the value of $\eta$ varies. The other parameter values are the same as the ones in Figure 8.

range $\eta$, vegetation patterns vary from spots to stripes. In particular, one can see that the short-range seed dispersal which corresponds to the large value of $\eta$ makes the width of vegetated region narrow while it makes the biomass density of vegetated region high.

The third case is on the seed germination rate. Figure 20 shows the effect of the seed germination rate $c$ on vegetation patterns. When the seed germination rate is quite small, vegetation patterns with very narrow width and very high density are formed $(c=0$ and 0.001$)$. As the value of $c$ increases, the width of vegetated region becomes thick and the biomass 


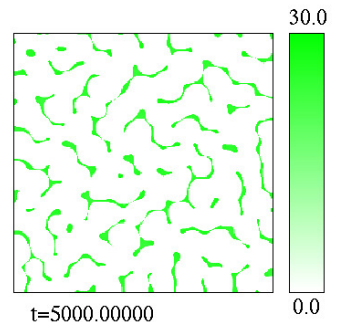

$c=0$

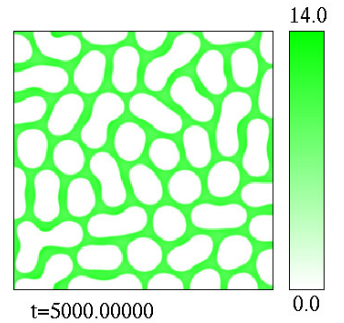

$c=0.2$

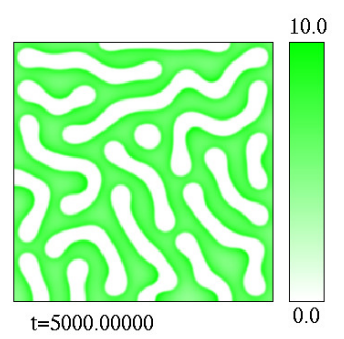

$c=0.8$

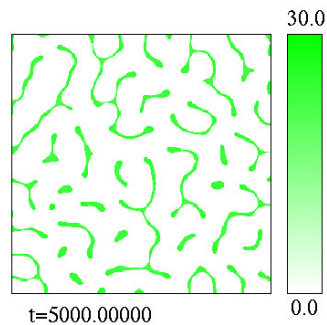

$c=0.001$

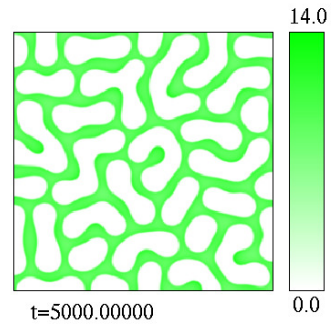

$c=0.3$

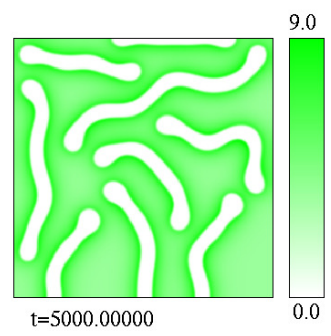

$c=1$

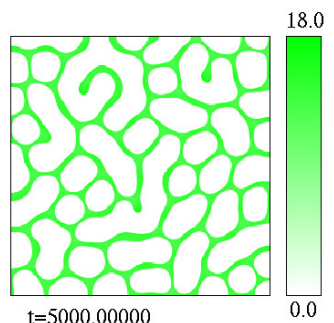

$c=0.05$

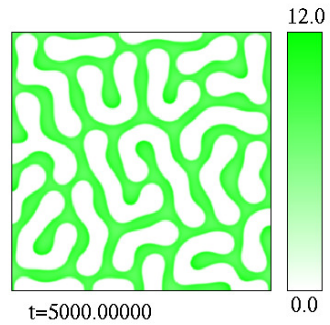

$c=0.4$

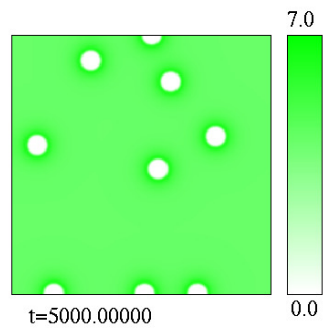

$c=1.4$

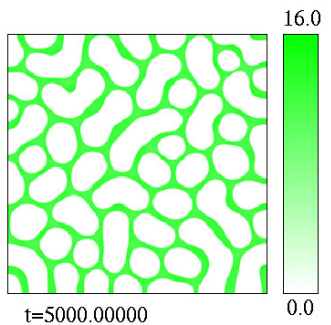

$c=0.1$

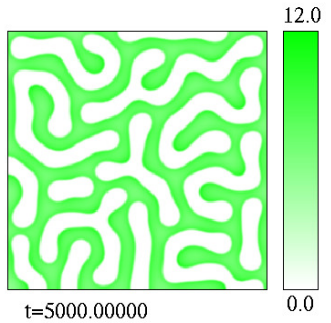

$c=0.6$

Figure 20: Vegetation patterns $P$ on a square domain $(0,15) \times(0,15)$ when the value of $c$ varies. The other parameter values are the same as the ones in Figure 10. 
density of vegetated region becomes low. And eventually uniform vegetation states are generated for $c=1.5$. As well as the one-dimensional case in Figure 10, the high germination rate results in uniform vegetation.

Finally, we show a transition of vegetation patterns when a kernel shape gradually changes from two-dimensional Gaussian function to a ring-shaped function whose peak is located away from the center, which corresponds to Figure 16 in the one-dimensional case. In other words, we use the two-dimensional version of the kernel (42) (see also Figure 12(b)) and change the value of $\zeta$ in the kernel. Numerical results according to the change of $\zeta$ are illustrated in Figure 21. As the peak of the kernel is apart from the center, that is, the value of $\zeta$ increases,

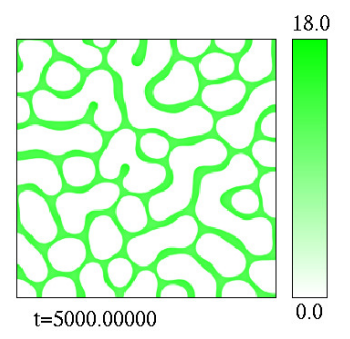

$$
\zeta=0
$$

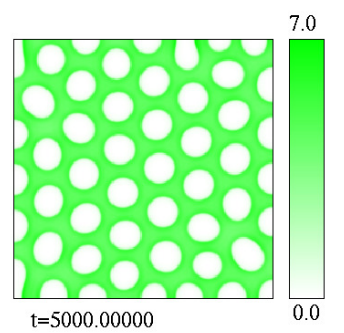

$\zeta=0.5$

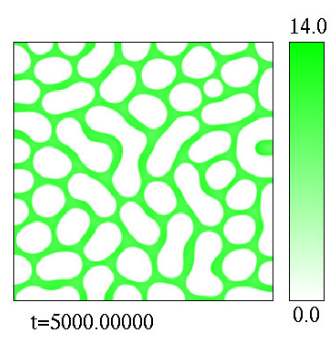

$$
\zeta=0.05
$$

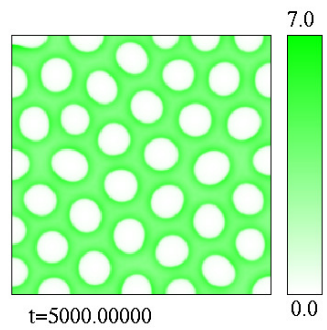

$\zeta=0.75$

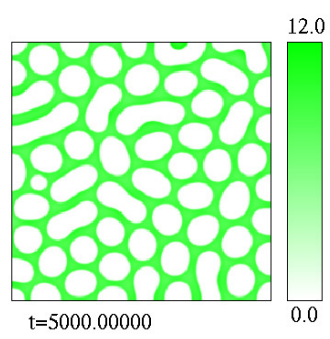

$\zeta=0.09$

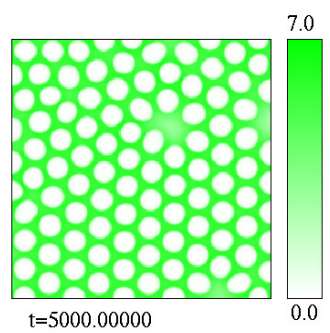

$\zeta=1.5$

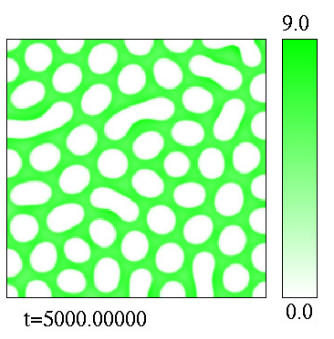

$$
\zeta=0.2
$$

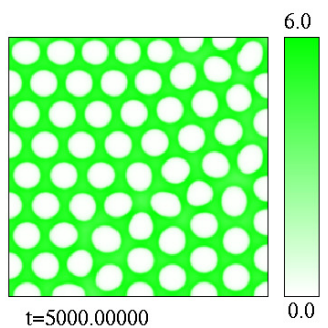

$\zeta=2$

Figure 21: Vegetation patterns $P$ on a square domain $(0,15) \times(0,15)$ when the value of $\zeta$ varies. The other parameter values are the same as the ones in Figure 16.

the patterns tend to change into regular gaps $(\zeta=0.5,0.75,1.5$ and 2$)$. These numerical results suggest that a strategy where plants spread the seeds far away (the peak of seed density is located away from the center) facilitates the formation of regular gap patterns.

\section{Concluding remarks}

In this paper, we have discussed a nonlocal system for vegetation in drylands: as argued in [27], in order to describe the plant dispersal, it is more reasonable to use a nonlocal term than to use a diffusion term. We have obtained the existence and uniqueness of a global solution in the nonlocal system, global structures of stationary solutions in the one-dimensional problem and relations between some parameters and two-dimensional patterns. The well-posedness is important as an estimate of the validity of the model. Also, when the kernel shape included in the nonlocal term changes, we investigated how it influences patterns. Although the nonlocal system discussed here is not a reaction-diffusion system, nonconstant vegetation patterns reminiscent of Turing patterns have been exhibited in a self-organized way. In this regard, 
understanding of the relation between a nonlocal system and a reaction-diffusion system is progressing [25].

We numerically demonstrated relations between four parameters in the system and vegetation patterns: the rainfall rate $r$, the seed dispersal range $\eta$, the seed germination rate $c$ and the peak distance of the seed density from the center $\zeta$. The relation between the rainfall rate and vegetation patterns has been discussed in several papers [7], [8], [10], [18], [27], [30], [38]. These papers reported that bare soil states, spot patterns, stripe patterns, gap patterns and uniform states are observed in turn according to an increase in the precipitation rate. However, the nonlocal system discussed in this paper exhibits labyrinth patterns for relatively large values of $r$, which possess wider vegetation stripes (see Figure 18). In other words, bare soil states, spot patterns, patterns with narrow stripes, gap patterns, patterns with wide stripes and uniform states are observed in this system according to an increase in the value of $r$. We note that the wide stripe patterns observed for relatively large values of $r$ are kept without drastic change even though the numerical computation continues furthermore. On the other hand, we showed a transition of vegetation patterns when the parameter $\eta$ varies in Figure 19. When the value of $\eta$ tends to infinity, we expect that $\int_{\Omega} P(t, y) \Phi(x, y) d y \rightarrow P(t, x)$. Then, the equation for $P$ in (2) turns into

$$
\frac{\partial P}{\partial t}=a \frac{W}{W+1} P-b P+c \frac{W}{W+1} P
$$

in this limiting situation. Therefore, it seems that a vegetation pattern when the value of $\eta$ tends to infinity possesses narrow width and high biomass density of vegetated regions like that for $c=0$ in Figure 20. Actually, one can see a tendency that the width of vegetated region becomes narrow and the biomass density of vegetated region becomes high depending on an increase in the value of $\eta$. However, the numerical simulation for $\eta>4000$ is very hard because of the spatial discretization.

Finally, we remark on the system (2) with the two-dimensional version of the kernel (42) which possesses a ring-shaped configuration for suitable $\zeta$ values. Interestingly, as shown in Figure 22, a strange and complicated pattern is generated when $\zeta=1$, which is completely different from regular gap patterns in Figure 21. Since such a pattern is never observed in

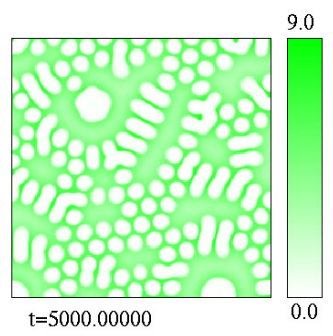

Figure 22: Vegetation patterns $P$ on a square domain $(0,15) \times(0,15)$ when $\zeta=1$. The other parameter values are the same as the ones in Figure 21.

the system (2) with a single peak kernel like a two-dimensional Gaussian function, it seems that the system (2) with a ring-shaped kernel includes rich mathematical structure from the viewpoint of pattern formation. Therefore, a strategy that plants spread the seeds far away may involve a variety of complicated vegetation patterns. Further investigation will be needed. 
We point out some controversial issues of the nonlocal model. As shown in Figure 3, the plant density $P$ linearly increases as the rainfall rate $r$ increases, which indicates that there is no saturation effect of the plant density in the system. In this point, the papers [20], [38] treat a model which takes into account ecological constraints. Moreover, the nonlocal model should include different time scales. For example, the time scale of water movement and infiltration should be different from that of plant growth, establishment etc. From these points of view, we think the model could be improved, and further analyses are required.

\section{Acknowledgements}

We are very grateful to the anonymous reviewers for very fruitful comments and suggestions, which really improved the quality of the paper. This work was supported by JSPS KAKENHI (HI: Grant Number 17K14237, MM: Grant Number 15K13462).

\section{References}

[1] E. J. Allen, L. J. S. Allen and X. Gilliam, Dispersal and competition models for plants, J. Math. Biol. 34 (1996) 455-481.

[2] F. Borgogno, P. D'Odorico, F. Laio and L. Ridolfi, Mathematical models of vegetation pattern formation in ecohydrology, Reviews of Geophysics 47 (2009) 2007RG000256.

[3] J. S. Clark, E. Macklin and L. Wood, Stages and spatial scales of recruitment limitation in southern Appalachian forests, Ecological Monographs 68 (1998) 213-235.

[4] V. Deblauwe, N. Barbier, P. Couteron, O. Lejeune and J. Bogaert, The global biogeography of semi-arid periodic vegetation patterns, Global Ecol. Biogeogr., 17 (2008) 715-723.

[5] E. J. Doedel, B. E. Oldeman, A. R. Champneys, F. Dercole, T. Fairgrieve, Y. Kuznetsov, R. Paffenroth, B. Sandstede, X. Wang and C. Zhang, AUTO-07P: Continuation and bifurcation software for ordinary differential equations.

[6] S. Getzin, H. Yizhaq, B. Bell, T. E. Erickson, A. C. Postle, I. Katra, O. Tzuk, Y. R. Zelnik, K. Wiegand, T. Wiegand and E. Meron, Discovery of fairy circle in Australia supports self-organization theory, Proc. Natl. Acad. Sci. U. S. A., 133 (2016) 3551-3556.

[7] E. Gilad, J. von Hardenberg, A. Provenzale, M. Shachak and E. Meron, Ecosystem engineers: from pattern formation to habitat creation, Phys. Rev. Lett. 93 (2004) 098105.

[8] E. Gilad, J. von Hardenberg, A. Provenzale, M. Shachak and E. Meron, A mathematical model of plants as ecosystem engineers, J. Theor. Biol., 244 (2007) 680-691.

[9] Y. Goto, D. Hilhorst, E. Meron and R. Temam, Existence theorem for a model of dryland vegetation, Discrete Contin. Dyn. Syst. Ser. B 16 (2011) 197-224.

[10] J. von Hardenberg, E. Meron, M. Shachak and Y. Zarmi, Diversity of vegetation patterns and desertification, Phys. Rev. Lett. 87 (2001) 198101.

[11] R. HilleRisLambers, M. Rietkerk, F. van den Bosch, H. H. T. Prins and H. de Kroon, Vegetation pattern formation in semi-arid grazing systems, Ecology 82 (2001) 50-61. 
[12] V. Hutson, S. Martinez, K. Mischaikow and G. T. Vickers, The evolution of dispersal, J. Math. Biol. 47 (2003) 483-517.

[13] B. J. Kealy and D. J. Wollkind, A nonlinear stability analysis of vegetative Turing pattern formation for an interaction-diffusion plant-surface water model system in an arid flat environment, Bull. Math. Biol. 74 (2012) 803-833.

[14] S. Kéfi, M. Rietkerk, C. L. Alados, Y. Pueyo, V. P. Papanastasis, A. ElAich and P. C. de Ruiter, Spatial vegetation patterns and imminent desertification in Mediterranean arid ecosystems, Nature 449 (2007) 213-217.

[15] S. Kinast, Y. R. Zelnik, G. Bel and E. Meron, Interplay between Turing mechanisms can increase pattern diversity, Phys. Rev. Lett. 112 (2014) 078701.

[16] C. A. Klausmeier, Regular and irregular patterns in semiarid vegetation, Science $\mathbf{2 8 4}$ (1999) 1826-1828.

[17] O. A. Ladyženskaja, V. A. Solonnikov and N. N. Ural'ceva, Linear and Quasilinear Equations of Parabolic Type, translated from the Russian by S. Smith. Translations of Mathematical Monographs, Vol. 23. American Mathematical Society, Providence, R.I., 1967.

[18] E. Meron, Modeling dryland landscapes, Math. Model. Nat. Phenom. 6 (2011) 163-187.

[19] E. Meron, Nolinear physics of ecosystems, CRC Press, 2015.

[20] E. Meron, Pattern formation -A missing link in the study of ecosystem response to environmental changes, Mathematical Biosciences 271 (2016) 1-18.

[21] E. Meron, E. Gilad, J. von Hardenberg, M. Shachak and Y. Zarmi, Vegetation patterns along a rainfall gradient, Chaos Solitons Fract. 19 (2004) 367-376.

[22] D. C. Mistro, L. A. D. Rodrigues and A. B. Schmid, A mathematical model for dispersal of an annual plant population with a seed bank, Ecological Modelling 188 (2005) 52-61.

[23] R. Nathan, U. N. Safriel, I. Noy-Meir and G. Schiller, Spatiotemporal variation in seed dispersal and recruitment near and far from Pinus halepensis trees, Ecology 81 (2000) $2156-2169$.

[24] M. G. Neubert, M. Kot and M. A. Lewis, Dispersal and pattern formation in a discretetime predator-prey model, Theoretical population biology, 48 (1995) 7-43.

[25] H. Ninomiya, Y. Tanaka and H. Yamamoto, Reaction, diffusion and non-local interaction, J. Math. Biol. 75 (2017) 1203-1233.

[26] T. Okayasu and Y. Aizawa, Systematic analysis of periodic vegetation patterns, Progress of Theoretical Physics 106 (2001) 705-720.

[27] Y. Pueyo, S. Kéfi, C. L. Alados and M. Rietkerk, Dispersal strategies and spatial organization of vegetation in arid ecosystems, Oikos 117 (2008) 1522-1532. 
[28] M. Rietkerk, P. Ketner, J. Burger, B. Hoorens and H. Olff, Multiscale soil and vegetation patchiness along a gradient of herbivore impact in a semi-arid grazing system in West Africa, Plant Ecology 148 (2000) 207-224.

[29] M. Rietkerk and J. van de Koppel, Regular pattern formation in real ecosystems, Trends in Ecology \& Evolution 23 (2008) 169-175.

[30] M. Rietkerk, S. C. Dekker, P. C. de Ruiter and J. van de Koppel, Self-organized patchiness and catastrophic shifts in ecosystems, Science 305 (2004) 1926-1929.

[31] M. Rietkerk, M. C. Boerlijst, F. van Langevelde, R. HilleRisLambers, J. van de Koppel, L. Kumar, H. H. T. Prins and A. M. de Roos, Self-organization of vegetation in arid ecosystems, The American Naturalist 160 (2002) 524-530.

[32] M. Scheffer, J. Bascompte, W. A. Brock, V. Brovkin, S. R. Carpenter, V. Dakos, H. Held, E. H. van Nes, M. Rietkerk and G. Sugihara, Early-warning signals for critical transitions, Nature 461 (2009) 53-59.

[33] J. A. Sherratt, When does colonisation of a semi-arid hillslope generate vegetation patterns?, J. Math. Biol. 73 (2016) 199-226.

[34] K. Siteur, E. Siero, M. B. Eppinga, J. D. M. Rademacher, A. Doelman and M. Rietkerk, Beyond Turing: The response of patterned ecosystems to environmental change, Ecological Complexity 20 (2014) 81-96.

[35] J. Smoller, Shock Waves and Reaction-Diffusion Equations, Springer-Verlag, New York, 1994.

[36] S. Thompson, G. Katul and S. M. McMahon, Role of biomass spread in vegetation pattern formation within arid ecosystems, Water Resources Research 44 (2008) W10421.

[37] C. V. Valentin, J. M. d'Herbès, J. Posen, Soil and water components of banded vegetation patterns, Catena 37 (1999) 1-24.

[38] Y. R. Zelnik, S. Kinast, H. Yizhaq, G. Bel and E. Meron, Regime shifts in models of dryland vegetation, Phil. Trans. R. Soc. A, 371 (2013) 20120358. 